\title{
Robust Distributed Fusion with Labeled Random Finite Sets
}

\author{
Suqi Li, Wei Yi*, Reza Hoseinnezhad, Giorgio Battistelli, Bailu Wang, Lingjiang Kong
}

\begin{abstract}
This paper considers the problem of the distributed fusion of multi-object posteriors in the labeled random finite set filtering framework, using Generalized Covariance Intersection (GCI) method. Our analysis shows that GCI fusion with labeled multi-object densities strongly relies on label consistencies between local multi-object posteriors at different sensor nodes, and hence suffers from a severe performance degradation when perfect label consistencies are violated. Moreover, we mathematically analyze this phenomenon from the perspective of Principle of Minimum Discrimination Information and the so called yesobject probability. Inspired by the analysis, we propose a novel and general solution for the distributed fusion with labeled multi-object densities that is robust to label inconsistencies between sensors. Specifically, the labeled multi-object posteriors are firstly marginalized to their unlabeled posteriors which are then fused using GCI method. We also introduce a principled method to construct the labeled fused density and produce tracks formally. Based on the developed theoretical framework, we present tractable algorithms for the family of generalized labeled multi-Bernoulli (GLMB) filters including $\delta$-GLMB, marginalized $\delta$-GLMB and labeled multi-Bernoulli filters. The robustness and efficiency of the proposed distributed fusion algorithm are demonstrated in challenging tracking scenarios via numerical experiments.
\end{abstract}

\section{INTRODUCTION}

D ISTRIBUTED multi-sensor multi-object tracking (DMMT) solutions are generally designed to benefit from lower communication cost and higher fault tolerance than centralized methods. Devising DMMT solutions becomes particularly challenging when the correlations between the estimates from different sensors are not known. The optimal solution to this problem was developed in [1], but the computational cost of calculating the common information can make the solution intractable in many real-world applications. A number of suboptimal solutions

This work was supported by the Chang Jiang Scholars Program, the National Natural Science Foundation of China under Grants 61501505 and 61771110, the Fundamental Research Funds of Central Universities under Grants ZYGX2016J031, the Chinese Postdoctoral Science Foundation under Grant 2014M550465 and Special Grant 2016T90845, and the Australian Research Council (ARC) through the Linkage Project Grant LP160101081. (Corresponding author: Wei Yi.)

S. Li, B. Wang, W. Yi, and L. Kong are with the School of Electronic Engineering, University of Electronic Science and Technology of China, Chengdu 611731, China (Email: qi_qi_zhu1210@163.com; kussoyi@gmail.com; w_b_13020@163.com; lingjiang.kong@gmail.com).

R. Hoseinnezhad is with the School of Aerospace, Mechanical and Manufacturing Engineering, RMIT University, Victoria 3083, Australia (Email: reza.hoseinnezhad@rmit.edu.au).

G. Battistelli is with the Dipartimento di Ingegneria dell' Informazione (DINFO), Università degli Studi di Firenze, Via Santa Marta 3, 50139, Firenze, Italy (Email: giorgio.battistelli@unifi.it). with demonstrated tractability have been formulated based on the Generalized Covariance Intersection (GCI) [2], [3] or the exponential mixture densities (EMD) [4]-[6] or the Kullback-Leibler average (KLA) [7], [8].

The GCI fusion rule is an extension of the Covariance Intersection method which only utilizes the mean and covariance and is limited to Gaussian posteriors [9]. The GCI fusion rule relaxes the Gaussian constraint, and can be used to fuse multiobject distributions with completely unknown correlations, since it intrinsically avoids any double counting of common information. Furthermore, the GCI can be computed in a distributed way by means of suitable consensus algorithms [7], [8]. Finally, from an information-theoretic point of view, GCI fusion rule admits a meaningful interpretation that the fused density is the centroid of the local posteriors with KullbackLeibler divergence considered as the distance.

Based on the GCI fusion rule and its variants, several DMMT algorithms have been proposed in the literature. Specifically, the distributed fusion algorithms for probability hypothesis density (PHD) [10], [11], cardinalized PHD (CPHD) [12], [13] and multi-Bernoulli (MB) [14]-[17] filters have been explored in [2], [5]-[8], [18]-[21]. The aforementioned methods are multi-object filters not trackers in the sense that object states are estimated without labels.

Recently, in a series of works, the notion of labeled random finite set (RFS) was introduced to address object trajectories and their uniqueness [22]-[28]. Vo et al. [22], [23] proposed a particular class of labeled multi-object densities called generalized labeled multi-Bernoulli (GLMB) densities ${ }^{1}$ The class of GLMB densities are conjugate priors with respect to the standard multi-object likelihood and also closed under the Chapman-Kolmogorov equation in Bayesian inference. A variant of the GLMB filter called the $\delta$-GLMB filter can be used to multi-object tracking directly, and not only produces trajectories formally but also outperforms the aforementioned filters [22]. Two computationally-efficient approximations of the $\delta$-GLMB filter, i.e., the labeled multi-Bernoulli (LMB) filter [24] and the marginalized $\delta$-GLMB (M $\delta$-GLMB) filter [26], have also been developed.

The enhanced accuracy and superior tracking capability that are inherent in the labeled random finite set filters, have motivated the development of distributed fusion methods that work in tandem with these multi-object tracking algorithms. Fantacci et al. [30] were among the first who investigated the distributed fusion of labeled multi-object densities and derived

\footnotetext{
${ }^{1}$ GLMB distribution was also named as Vo-Vo distribution by Mahler in his book [29].
} 
closed-form solutions for GCI fusion with M $\delta$-GLMB and LMB densities, based on the assumption that different sensors share the same label space for the birth process. However, their work does not explore the profound meaning of "sharing the same label space" and does not address the conditions implied by the assumption in practice.

In this paper, we further investigate the distributed fusion for labeled random finite set filters. Our major contributions are as follow:

i) We analyse the drawback of GCI fusion with labeled multi-object densities by showing that the fusion performance is highly sensitive to label inconsistencies between sensor nodes. The analysis is carried out in a principled theoretical framework by virtue of the following proposed notions:

- GCI divergence, which is a new measure of discrepancy compatible with GCI fusion rule to quantify the degree of similarity between multiple densities.

- Conditional multi-label distribution, which facilitates a new decomposition of the labeled multi-object density.

- Label inconsistency indicator, which quantifies the inconsistency of label information embedded in multiple labeled multi-object densities.

ii) Motivated by the aforementioned performance analysis, we propose a novel and general solution to the distributed fusion with labeled set filters that is immune to the effect of label inconsistencies between sensor nodes. Based on the developed theoretical framework, we also present tractable distributed fusion algorithms for the family of GLMB filters including the $\delta$-GLMB, M $\delta-G L M B$ and LMB filters.

Extensive numerical experiments verify the robustness and effectiveness of the proposed fusion algorithm with Gaussian mixture implementation in challenging tracking scenarios.

Preliminary results have been announced in the conference paper [31]. This paper presents a more complete theoretical and numerical study. The layout of this paper is as follows. The background and notations are presented in Section III followed by drawbacks of GCI fusion with labeled densities discussed in Section III Section IV provides a mathematical analysis of the performance degradation of GCI fusion with labeled densities. Section $\mathrm{V}$ proposes a robust solution to distributed fusion with labeled set filters and presents tractable algorithms for the family of GLMB filters. Section VI demonstrates the performance of the proposed algorithms via numerical examples. Conclusions are presented in Section VII.

\section{NOTATIONS AND BACKGROUND}

\section{A. Notations}

We adopt the convention that single-object states are denoted by lowercase letter "x", e.g. $x, \mathrm{x}$, while the multiobject states are denoted by capital letter "X", e.g. $X, \mathbf{X}$. To distinguish labeled states and distributions from the unlabeled ones, bold face letters are adopted for the labeled ones, e.g. $\mathbf{x}, \mathbf{X}, \boldsymbol{\pi}$. Observations generated by single-object states are denoted by $z$, and the multi-object observations are denoted by $Z$. Moreover, blackboard bold letters represent spaces, e.g. the state space is represented by $\mathbb{X}$, the label space by $\mathbb{L}$, and the observation space by $\mathbb{Z}$. The collection of all finite subsets of $\mathbb{X}$ is denoted by $\mathcal{F}(\mathbb{X})$. The number of elements in a set is called its cardinality, and denoted by $|\cdot|$. The set of all finite subsets of $\mathbb{X}$ with cardinality $n$ is denoted by $\mathcal{F}_{n}(\mathbb{X})$.

We use the multi-object exponential notation

$$
h^{X} \triangleq \prod_{x \in X} h(x)
$$

for any set $X$ and real-valued function $h$, with $h^{\emptyset}=1$ by convention. The inclusion function is given by

$$
1_{Y}(X) \triangleq\left\{\begin{array}{l}
1, \quad \text { if } X \subseteq Y \\
0 \text { otherwise }
\end{array}\right.
$$

If $X$ is a singleton, i.e., $X=\{x\}$, the notation $1_{Y}(x)$ is used instead of $1_{Y}(\{x\})$.

\section{B. Labeled Random Finite Set Distributions and Filters}

Let $\mathcal{L}: \mathbb{X} \times \mathbb{L} \rightarrow \mathbb{L}$ be the projection defined by $\mathcal{L}((x, \ell))=$ $\ell$, then $\mathcal{L}(\mathbf{x})$ is called the label of the point $\mathbf{x} \in \mathbb{X} \times \mathbb{L}$. A finite subset $\mathbf{X}$ of $\mathbb{X} \times \mathbb{L}$ is said to have distinct labels if and only if $\mathbf{X}$ and its labels $\mathcal{L}(\mathbf{X}) \triangleq\{\mathcal{L}(\mathbf{x}): \mathbf{x} \in \mathbf{X}\}$ have the same cardinality. We define the distinct label indicator of $\mathbf{X}$ as $\Delta(\mathbf{X})=\delta_{|\mathbf{X}|}(|\mathcal{L}(\mathbf{X})|)$.

Definition 1. Given a labeled multi-object density $\pi$ on $\mathbb{X} \times$ $\mathbb{L}$, and any positive integer $n$, we define the joint existence probability of the label set $\left\{\ell_{1}, \cdots, \ell_{n}\right\} \subseteq \mathbb{L}$ by

$w\left(\left\{\ell_{1}, \cdots, \ell_{n}\right\}\right) \triangleq \int \boldsymbol{\pi}\left(\left\{\left(x_{1}, \ell_{1}\right), \cdots,\left(x_{n}, \ell_{n}\right)\right\}\right) d\left(x_{1}, \cdots, x_{n}\right)$

and the joint probability density on $\mathbb{X}^{n}$ conditional on their corresponding labels $\ell_{1}, \cdots, \ell_{n}$ by

$$
p\left(\left\{\left(x_{1}, \ell_{1}\right), \cdots,\left(x_{n}, \ell_{n}\right)\right\}\right) \triangleq \frac{\boldsymbol{\pi}\left(\left\{\left(x_{1}, \ell_{1}\right), \cdots,\left(x_{n}, \ell_{n}\right)\right\}\right)}{w\left(\left\{\ell_{1}, \cdots, \ell_{n}\right\}\right)}
$$

For $n=0$, we define $w(\emptyset) \triangleq \boldsymbol{\pi}(\emptyset)$ and $p(\emptyset) \triangleq 1$. It is implicit that $p(\mathbf{X})$ is defined to be zero whenever $w(\mathcal{L}(\mathbf{X}))$ is zero.

Definition 1 is first provided in [32], and using Definition 1 , the labeled multi-object density can be decomposed as

$$
\boldsymbol{\pi}(\mathbf{X})=w(\mathcal{L}(\mathbf{X})) p(\mathbf{X}) .
$$

In this paper, we focus on two most commonly used labeled multi-object distributions, namely the GLMB and LMB distributions. A GLMB labeled RFS is distributed according to: [22], [29]

$$
\boldsymbol{\pi}(\mathbf{X})=\Delta(\mathbf{X}) \sum_{c \in \mathbb{C}} w^{(c)}(\mathcal{L}(\mathbf{X}))\left[p^{(c)}\right]^{\mathbf{X}}
$$

where $\mathbb{C}$ is a discrete index set, and $w^{(c)}(L)$ and $p^{(c)}$ satisfy

$$
\sum_{L \subseteq \mathbb{L}} \sum_{c \in \mathbb{C}} w^{(c)}(L)=1 \text {, and } \int p^{(c)}(x, \ell) d x=1 .
$$

A GLMB RFS is completely characterized by the set of parameters $\left\{\left(\omega^{(c)}(I), p^{(c)}(\cdot)\right):(I, c) \in \mathcal{F}(\mathbb{L}) \times \mathbb{C}\right\}$.

A labeled multi-Bernoulli (LMB) RFS [24] with state space $\mathbb{X}$, label space $\mathbb{L}$ and (finite) parameter set $\left\{\left(r^{(\ell)}, p^{(\ell)}(x)\right)\right.$ : $\ell \in \mathbb{L}\}$, is distributed according to

$$
\boldsymbol{\pi}(\mathbf{X})=\Delta(\mathbf{X}) w(\mathcal{L}(\mathbf{X})) p^{\mathbf{X}}
$$


where

$$
\begin{aligned}
w(I) & =\prod_{i \in \mathbb{L}}\left(1-r^{(i)}\right) \prod_{\ell \in I} 1_{\mathbb{L}}(\ell) \frac{r^{(\ell)}}{1-r^{(\ell)}} \\
p(x, \ell) & =p^{(\ell)}(x) .
\end{aligned}
$$

The centerpiece of the RFS based multi-object filtering is the Bayes multi-object filter [33], which recursively propagates the multi-object posterior density forward in time through a prediction then an update step. When the objects are modelled by labeled RFSs, the Bayesian filter also becomes a multiobject tracker, as object identities (along with other parameters of labeled multi-object distributions) are propagated through the prediction and update steps of the filter. Of particular interest in this paper is the $\delta$-GLMB filter proposed by Vo et al. [22], [23]. This filter is devised based on assuming a special type of GLMB distribution (called $\delta$-GLMB distribution), and is more intuitive on label and data association hypotheses and can be directly implemented.

Due to presence of explicit data associations in the standard multi-object likelihood, the $\delta$-GLMB suffers from exponential growth of number of components of the posterior with time. To resolve this problem, approximations of $\delta$-GLMB filter that allow tractability have been proposed. An approximation that preserves both the first-order moment and cardinality distribution of the posterior is the M $\delta$-GLMB filter [26]. A faster yet less accurate approximation (that only preserves the first-order moment of the posterior) is LMB filter [24]. The LMB filter can be implemented substantially faster than the M $\delta$-GLMB filter.

Remark 1. In this paper, an RFS $X$ defined on space $\mathcal{F}(\mathbb{X})$ is referred to as an unlabeled RFS, while an RFS $\mathbf{X}$ defined on space $\mathcal{F}(\mathbb{X} \times \mathbb{L})$ with each realization having distinct labels is referred to as a labeled RFS. Both unlabeled and labeled RFSs belong to the family of simple finite point processes [34].

\section{Generalized Covariance Intersection (GCI)}

Using the concept of GCI for distributed multi-sensor fusion was first proposed by Mahler [2] who later developed the GCI fusion to extend the theory of finite set statistics (FISST) to the distributed environment. Consider a set of sensor nodes $\mathcal{N}=$ $\left\{1,2, \cdots, N_{s}\right\}$ in a sensor network. Suppose that in each node $s \in \mathcal{N}$, an RFS-based multi-object filter returns a local multiobject posterior $\pi_{s}(X)$ defined on a space $\chi$ and computed on the basis of the local information embedded in measurements acquired at node $s$. The GCI fusion rule combines all the local posteriors, returning their geometric mean in the form of an exponential mixture of the local densities,

$$
\pi_{\omega}(X)=\frac{\prod_{s \in \mathcal{N}}\left[\pi_{s}(X)\right]^{\omega_{s}}}{\int \prod_{s \in \mathcal{N}}\left[\pi_{s}(X)\right]^{\omega_{s}} \delta X}
$$

where the integral is a set integral as defined in FISST (see [33]) and the weights $\omega_{s}$ are user-defined parameters with the constraint:

$$
\omega_{s} \geqslant 0, \quad \sum_{s \in \mathcal{N}} \omega_{s}=1 .
$$

Note that the GCI rule 9 ) can also be used to fuse labeled multi-object densities. In that case, the labeled set integral should be used, as defined in [23]. With labeled multi-object posteriors the space $\chi$ is $\mathbb{X} \times \mathbb{L}$, otherwise we have $\chi=\mathbb{X}$.

The name GCI stems from the fact that 9 ) is the multiobject counterpart of the analogous fusion rule for (singleobject) probability densities [4] which, in turn, is a generalization of Covariance Intersection originally conceived for Gaussian probability densities [9]. In [7], [35], it has been shown that the GCI fusion in 9 essentially minimizes the weighted sum of the Kullback-Leibler divergence (KLD) with respect to the local densities, i.e.

$$
\pi_{\omega}=\arg \min _{\pi} \sum_{s \in \mathcal{N}} \omega_{s} D_{\mathrm{KL}}\left(\pi ; \pi_{s}\right)
$$

where $D_{\mathrm{KL}}$ denotes the KLD, defined as:

$$
D_{\mathrm{KL}}(f ; g) \triangleq \int f(X) \log (f(X) / g(X)) \delta X .
$$

In view of (11), the GCI fusion is also called KullbackLeibler average (KLA) fusion. In Bayesian statistics, the KLD can be seen as the information gain achieved when moving from a prior density to a posterior density. Hence, the GCI fusion essentially provides the density that minimizes the weighted sum of the information gains from the local densities on the basis of (11). This choice is coherent with the Principle of Minimum Discrimination Information (PMDI) according to which the probability density which best represents the current state of knowledge is the one which produces an information gain as small as possible. This property is important in order to ensure immunity to double counting, thus avoiding being overconfident on the available information.

\section{DRAWbacks of GCI Fusion With LABELED Multi-ObJect Densities}

Consider a set of labeled multi-object densities and the corresponding weights $\boldsymbol{\Pi}=\left\{\left(\boldsymbol{\pi}_{s}(\mathbf{X}), \omega_{s}\right)\right\}_{s \in \mathcal{N}}$, with each $\pi_{s}$ defined on space $\mathbb{X} \times \mathbb{L}$, in the form of $(3)$,

$$
\begin{aligned}
& \boldsymbol{\pi}_{s}\left(\left\{\left(x_{1}, \ell_{1}\right), \cdots,\left(x_{n}, \ell_{n}\right)\right\}\right)= \\
& \quad w_{s}\left(\left\{\ell_{1}, \cdots, \ell_{n}\right\}\right) p_{s}\left(\left\{\left(x_{1}, \ell_{1}\right), \cdots,\left(x_{n}, \ell_{n}\right)\right\}\right) .
\end{aligned}
$$

Substituting into the GCI fusion rule (9), leads to a fused density in the similar form

$$
\begin{aligned}
& \boldsymbol{\pi}_{\omega}\left(\left\{\left(x_{1}, \ell_{1}\right), \cdots,\left(x_{n}, \ell_{n}\right)\right\}\right)= \\
& w_{\omega}\left(\left\{\ell_{1}, \cdots, \ell_{n}\right\}\right) p_{\omega}\left(\left\{\left(x_{1}, \ell_{1}\right), \cdots,\left(x_{n}, \ell_{n}\right)\right\}\right),
\end{aligned}
$$

where

$$
\begin{aligned}
& w_{\omega}\left(\left\{\ell_{1}, \cdots, \ell_{n}\right\}\right)= \\
& \frac{\prod_{s \in \mathcal{N}}\left[w_{s}\left(\left\{\ell_{1}, \cdots, \ell_{n}\right\}\right)\right]^{\omega_{s}} \eta\left(\left\{\ell_{1}, \cdots, \ell_{n}\right\}\right)}{\sum_{I \in \mathcal{F}(\mathbb{L})} \prod_{s \in \mathcal{N}}\left[w_{s}(I)\right]^{\omega_{s}} \eta(I)} \\
& p_{\omega}\left(\left\{\left(x_{1}, \ell_{1}\right), \cdots,\left(x_{n}, \ell_{n}\right)\right\}\right)= \\
& \frac{\prod_{s \in \mathcal{N}}\left[p_{s}\left(\left\{\left(x_{1}, \ell_{1}\right), \cdots,\left(x_{n}, \ell_{n}\right)\right\}\right)\right]^{\omega_{s}}}{\eta\left(\left\{\ell_{1}, \cdots, \ell_{n}\right\}\right)}
\end{aligned}
$$

with

$$
\begin{aligned}
\eta\left(\left\{\ell_{1}, \cdots, \ell_{n}\right\}\right)=\int \prod_{s \in \mathcal{N}}\left[p_{s}\left(\left\{\left(x_{1}, \ell_{1}\right), \cdots,\left(x_{n}, \ell_{n}\right)\right\}\right)\right]^{\omega_{s}} \\
\\
d\left(x_{1} \cdots x_{n}\right) .
\end{aligned}
$$


This indicates that GCI fusion for labeled densities essentially is performed label-wise, and thus inherently demands perfect label consistencies between different local sensors, i.e., the same track has the same label in all sensor nodes. When different labels are associated with the same object in different sensors, GCI fusion does not make sense and indeed performs poorly because the probabilities $w_{s}(I)$ or the conditional probability density $p_{s}(\mathbf{X})$ for the same label set hypothesis (except for $\emptyset$ ) can have large disparity at different sensor nodes, which can largely diminish the fused probability of the label set hypothesis, $w_{\omega}(I)$.

In the following, we present three common phenomena that lead to the label consistency assumption being violated and GCI fusion failing to produce accurate results.

\section{Using Adaptive Birth Processes}

The standard formulation of labeled multi-object filters is based on assuming that object birth process is known as a priori. In some practical situations where the objects can appear anywhere in the surveillance area, the object birth intensity needs to cover the entire the surveillance area and does not add any prior information to the filtering process. An extension which can distinguish between the persistent and newly-born objects is to formulate an adaptive birth process that is tuned at each scan using the received observations. In presence of such adaptive birth processes at each sensor of a distributed multi-object tracking system, the same newly-born object may be labeled differently in different sensors.

\section{Random Uncertainties in Observations}

Suppose different sensors share the same prior information based birth processes [23]. In this situation, even if the same label drawn from the label spaces of different sensors has the same implication, statistical distribution of labels conditioned on the observation set could be substantially different from one sensor node to another, because of randomness of observations. As a result, the estimated label for the same object may be different in different sensor nodes.

Note that to ensure uniqueness of labels, in labeled multiobject tracking algorithms, a label $\ell$ is comprised of two elements: the time of birth $k$ and the index $i$ that distinguishes different objects born at the same time, i.e. $\ell=(k, i)$. It is common that with the labeled multi-object posterior formed in each sensor node, for each object there is a label, say $(\hat{k}, \hat{i})$, with a large weight and hence, representing the estimated label for that object. Due to false alarms (clutters), miss-detections or excessive observation noise, the estimated time of birth $\hat{k}$ may be different from one sensor node to another and from the true time of birth $k_{0}$. Specifically,

- $\hat{k}<k_{0}$ may occur in a local sensor node due to a false measurement appearing nearby the true track before $k_{0}$, leading to the deduction that the object is born earlier than $k_{0}$.

- $\hat{k}>k_{0}$ may occur in a local sensor node due to the excessive observation noise or mis-detection of the object during a few first time steps, leading to the deduction that the object is born after $k_{0}$.

\section{Local pruning}

For the sake of numerical tractability of the labeled set filtering algorithms, and reduction of communication costs in a distributed sensor network, pruning strategies are usually devised to keep the number of hypotheses bounded in each sensor node. This can clearly lead to an object's label to be pruned in one sensor node while remaining in the other, and the label-wise GCI fusion will lead to that the fused label set hypothesis including this label to be given a zero probability because it not supported by all sensor nodes.

Example 1. Consider a sensor network with two sensors employing an LMB filter in each sensor node. The surveillance region is $[-800,800] \mathrm{m} \times[-600,600] \mathrm{m}$. The standard dynamic and observation models provided in [23] are used. The observation model of each single object is linear Gaussian, the probability of detection $P_{D, k}=0.99$, and the intensity function of clutter $\kappa(\cdot)=5.2 \times 10^{-6}$. The transition of each single target follows the linear Gaussian model, and the probability of survival $P_{S, k}=0.99$. Both sensors have the prior knowledge that objects are born during times $k=4 \mathrm{~s}, 5 \mathrm{~s}$ or $6 \mathrm{~s}$, and around the origin $(0,0) \mathrm{m}$. The birth process used at each sensor is a labeled Bernoulli process $\left(r^{(k, i)}, p^{(k, i)}(x)\right)$,

$$
p^{(k, i)}(x)=\mathcal{N}(x ;(0,0,0,0) ; \operatorname{diag}([300300300300]))
$$

where the state $x$ is a vector of planar position and velocity, the index $i=1$ because only one object is born at the same time, and $k$ denotes the time of birth taking values from $\{4,5,6\} \mathrm{s}$. The true object is born at $k=5 \mathrm{~s}$, and the true track is shown in Fig. 1 (a). Two representative types of measurements from sensors 1 and 2 are shown in Fig. 1 (a). No pruning strategies are adopted.

Fig. 1 (a) also shows object state estimates computed at each sensor node of a single run, which reflect that both local sensors can estimate the kinematic states accurately in general. However, the time of birth estimates computed at the two sensor nodes have a small but indeed existing difference. Specifically,

Sensor 1: due to an excessively noisy measurement at $k=5 \mathrm{~s}$, the object is missed but detected for the first time at $\hat{k}_{1}=6 \mathrm{~s}$ which is later than the true time of birth.

Sensor 2: at time $k=4 \mathrm{~s}$ a false measurement is close to the true track and lead to an estimated birth time of $\hat{k}_{2}=4 \mathrm{~s}$ which is earlier than the true time of birth.
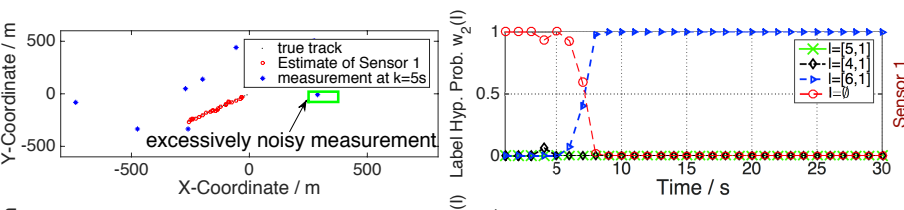

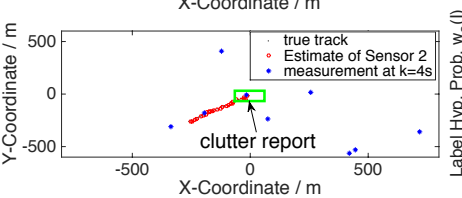

(a)

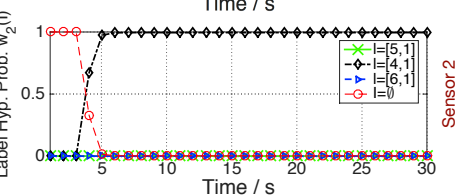

(b)
Fig. 1. (a) Tracks and measurements at two sensors; (b) The respective posterior probabilities $w_{1}(I)$ and $w_{2}(I)$ for different label set hypotheses at sensors 1 and 2 . 
Fig. 1 (b) shows the posterior probabilities $w_{1}(I)$ and $w_{2}(I)$ for different label set hypotheses $I=\{(4,1)\},\{(5,1)\},\{(6,1)\}, \emptyset$ at sensor nodes 1 and 2 , respectively. A large disparity can be observed between the probabilities of the same label set hypothesis at the two sensor nodes, which is the main reason why $\hat{k}_{1}$ and $\hat{k}_{2}$ are different. Furthermore, if we prune all the label set hypotheses with probabilities less than a threshold $\Gamma=10^{-6}$, at times $k>8 \mathrm{~s}$, only one label set hypothesis survives at each sensor node: $(6,1)$ at sensor 1 , and $(4,1)$ at sensor 2 . GCI fusion will be completely erroneous in this case.

\section{Motivating Analysis}

In the previous section, we revealed the drawback of GCI fusion for labeled multi-object densities through an intuitive observation of the fusion formulas, three common phenomena and a typical numerical experiment. In this section, we present a principled analysis of how label inconsistencies affect the fusion performance from the perspective of PMDI and the declaration of the object existence. The performance analysis will also motivate the proposed solution that is robust to label mismatches.

\section{A. Mathematical Tools}

We firstly introduce the following notions which are the basis of the subsequent theoretical analyses.

1) Conditional Multi-label Distribution: We firstly review the mathematical treatment to transform a labeled multi-object density to its unlabeled version. The unlabeled version of a labeled RFS on $\mathbb{X} \times \mathbb{L}$ is given by $\mathcal{K}(\mathbf{X})=\{\mathcal{K}(\mathbf{x}): \mathbf{x} \in$ $\mathbf{X}\}$, where $\mathcal{K}: \mathbb{X} \times \mathbb{L} \rightarrow \mathbb{X}$ is the projection defined by $\mathcal{K}((x, \ell))=x$. Given a labeled RFS $\mathbf{X}$ distributed according to $\pi, X=\mathcal{K}(\mathbf{X})$ is distributed according to the following marginal: [23]

$\pi\left(\left\{x_{1}, \cdots, x_{n}\right\}\right)=\sum_{\left(\ell_{1}, \cdots, \ell_{n}\right) \in \mathbb{L}^{n}} \pi\left(\left\{\left(x_{1}, \ell_{1}\right), \cdots,\left(x_{n}, \ell_{n}\right)\right\}\right)$.

Definition 2. Given a labeled multi-object density $\boldsymbol{\pi}$ on $\mathbb{X} \times \mathbb{L}$, and any positive integer $n$, the joint probability distribution of labels $\ell_{1}, \cdots, \ell_{n}$ on $\mathbb{L}^{n}$ conditional on their corresponding (unlabeled) states $x_{1}, \cdots, x_{n}$ is given by

$\varpi\left(\left\{\left(\ell_{1} \mid x_{1}\right), \cdots,\left(\ell_{n} \mid x_{n}\right)\right\}\right) \triangleq \frac{\boldsymbol{\pi}\left(\left\{\left(x_{1}, \ell_{1}\right), \cdots,\left(x_{n}, \ell_{n}\right)\right\}\right)}{\pi\left(\left\{x_{1}, \cdots, x_{n}\right\}\right)}$

where $\pi\left(\left\{x_{1}, \cdots, x_{n}\right\}\right)$ is given in $(17)$. By convention, $\varpi(\emptyset) \triangleq 1$.

Note that (18) can be rewritten as:

$$
\begin{aligned}
& \boldsymbol{\pi}\left(\left\{\left(x_{1}, \ell_{1}\right), \cdots,\left(x_{n}, \ell_{n}\right)\right\}\right)= \\
& \varpi\left(\left\{\left(\ell_{1} \mid x_{1}\right), \cdots,\left(\ell_{n} \mid x_{n}\right)\right\}\right) \pi\left(\left\{x_{1}, \cdots, x_{n}\right\}\right)
\end{aligned}
$$

where $n$ takes values from the field of real number $\mathbb{N}$, which actually provides a new decomposition of labeled multiobject density. Thus, the conditional multi-label distribution $\varpi\left(\left\{\left(\ell_{1} \mid x_{1}\right), \cdots,\left(\ell_{n} \mid x_{n}\right)\right\}\right)$ encapsulates all label-related information embedded in the labeled multi-object density $\boldsymbol{\pi}(\cdot)$. It is this information that makes it possible to estimate the labels of kinematic states and produce tracks in labeled set filters.
2) GCI Divergence: In this subsection, GCI divergence is introduced as a new measure of discrepancy to quantify the degree of similarity between multiple densities, and evaluate the minimal information gain of GCI fusion with multiple densities. Consider a set of multi-object densities associated with their corresponding weights, denoted by

$$
\Pi=\left\{\left(\pi_{s}(X), \omega_{s}\right): s \in \mathcal{N}\right\}
$$

where each $\pi_{s}(X)$ is defined on the same space $\chi$, each weight $\omega_{s}$ is a given confidence for $\pi_{s}$, and the weights satisfy (10). For any multi-object density $\pi(X)$ on $\chi$ (possibly $\mathbb{X}$ or $\mathbb{X} \times$ $\mathbb{L}$ ), the weighted average information gain (AIG) from $\pi$ to densities in $\Pi$ is defined as

$$
D_{\mathrm{AIG}}(\pi ; \Pi) \triangleq \sum_{s \in \mathcal{N}} \omega_{s} D_{\mathrm{KL}}\left(\pi ; \pi_{s}\right)
$$

According to (11), the GCI fusion rule by principle minimizes the weighted AIG. The resulting minimal weighted AIG over all densities on $\chi$ is given by: [7]

$$
\min _{\pi} D_{\mathrm{AIG}}(\pi ; \Pi)=-\log c(\Pi)
$$

where

$$
c(\Pi)=\int \prod_{s \in \mathcal{N}}\left[\pi_{s}(X)\right]^{\omega_{s}} \delta X .
$$

The quantity $c(\Pi)$ is referred to as GCI coefficient. It is always in $[0,1]$ and presents a measure of similarity between densities in $\Pi$. In this paper, we will call the minimal weighted AIG given in 21) and (22) as GCI divergence denoted by $G(\Pi)$ :

$$
G(\Pi)=-\log c(\Pi)=-\log \int \prod_{s \in \mathcal{N}}\left[\pi_{s}(X)\right]^{\omega_{s}} \delta X .
$$

GCI divergence is a tool to quantify the degree of similarity between multiple densities. The larger the GCI divergence among densities in $\Pi$ is, the more the corresponding GCI fusion is violating the PMDI. Hence a large GCI divergence can be an indication that the information contained in the densities to be fused are not coherent. The extreme case $G(\Pi) \rightarrow+\infty\left(c(\Pi) \rightarrow 0^{+}\right)$occurs when the densities are compeletly incompatible and have different supports.

3) Yes-Object Probability: We define yes-object probability and no-object probability as $P_{\mathrm{y}}(\pi) \triangleq 1-\pi(\emptyset)$ and $P_{\mathrm{n}}(\pi) \triangleq$ $\pi(\emptyset)$, respectively, for a given multi-object posterior $\pi$ [33]. Usually, object existence can be declared only if the yes-object probability is greater than a threshold $\tau$ ( usually $0.5 \leqslant \tau<1$ ). The yes-object probability is the basis of multi-object state estimation in the sense that only if the existence of objects can be declared, the object states can be extracted; otherwise the best estimate of multi-object state set will be an empty set (no-object inference).

\section{B. Theoretical Analysis}

This section provides a thorough theoretical analysis of how the label inconsistencies between different labeled multi-object densities affect the performance of GCI fusion.

Consider a set of labeled multi-object densities $\Pi=$ $\left\{\left(\boldsymbol{\pi}_{s}, \omega_{s}\right)\right\}_{s \in \mathcal{N}}$ with each $\boldsymbol{\pi}_{s}$ defined on space $\mathbb{X} \times \mathbb{L}$, and a set of unlabeled multi-object densities $\Pi=\left\{\left(\pi_{s}, \omega_{s}\right)\right\}_{s \in \mathcal{N}}$ with each $\pi_{s}$ the marginal of $\pi_{s}$ on $\mathbb{X}$. The following proposition 
states the relationship between GCI divergences of the two sets of multi-object densities.

Proposition 1. If each labeled multi-object density $\boldsymbol{\pi}_{s}(\cdot)$ is

$$
\begin{aligned}
& \boldsymbol{\pi}_{s}\left(\left\{\left(x_{1}, \ell_{1}\right), \cdots,\left(x_{n}, \ell_{n}\right)\right\}\right)= \\
& \quad \varpi_{s}\left(\left\{\left(\ell_{1} \mid x_{1}\right), \cdots,\left(\ell_{n} \mid x_{n}\right)\right\}\right) \pi_{s}\left(\left\{x_{1}, \cdots, x_{n}\right\}\right)
\end{aligned}
$$

of form (19), then the GCI divergence for densities in $\Pi$ is given by

$$
G(\boldsymbol{\Pi})=G(\Pi)-\log \mathrm{E}_{\pi_{\omega}}[\mu(X)]
$$

where $G(\Pi)$ is the GCI divergence for densities in $\Pi$, and

$$
\sum_{\left(\ell_{1}, \cdots, \ell_{n}\right) \in \mathbb{L}^{n}} \prod_{s \in \mathcal{N}^{\prime}}\left[\varpi_{s}\left(\left\{\left(\ell_{1} \mid x_{1}\right), \cdots,\left(\ell_{n} \mid x_{n}\right)\right\}\right)\right]^{\omega_{s}}
$$

is GCI coefficient for the set of conditional multi-label distributions $\left\{\left(\varpi_{s}\left(\left\{\left(\ell_{1} \mid x_{1}\right), \cdots,\left(\ell_{n} \mid x_{n}\right)\right\}\right), \omega_{s}\right)\right\}_{s \in \mathcal{N}}$, and $\mathrm{E}_{\pi_{\omega}}(\cdot)$ denotes expectation with respect to $\pi_{\omega}$, with $\pi_{\omega}$ being the fused density returned by GCI fusion of all densities in $\Pi$.

Proof. Substituting the densities in (24) in the definition of GCI divergence 23, leads to

$$
\begin{gathered}
G(\boldsymbol{\Pi})=-\log \sum_{n=0}^{\infty} \frac{1}{n !} \int \prod_{s \in \mathcal{N}^{[}}\left[\pi_{s}\left(\left\{x_{1}, \cdots, x_{n}\right\}\right)\right]^{\omega_{s}} \\
\sum_{\left(\ell_{1}, \cdots, \ell_{n}\right) \in \mathbb{L}^{n}} \prod_{s \in \mathcal{N}}\left[\varpi_{s}\left(\left\{\left(\ell_{1} \mid x_{1}\right), \cdots,\left(\ell_{n} \mid x_{n}\right)\right\}\right)\right]^{\omega_{s}} d\left(x_{1}, \cdots, x_{n}\right) .
\end{gathered}
$$

Substituting

$$
c(\Pi)=\sum_{n=0}^{\infty} \frac{1}{n !} \int \prod_{s \in \mathcal{N}}\left[\pi_{s}\left(\left\{x_{1}, \cdots, x_{n}\right\}\right)\right]^{\omega_{s}} d\left(x_{1}, \cdots, x_{n}\right)
$$

and 26) into 27,,$G(\boldsymbol{\Pi})$ can be rewritten as

$$
\begin{aligned}
G(\boldsymbol{\Pi})= & -\log c(\Pi) \sum_{n=0}^{\infty} \frac{1}{n !} \int \frac{\prod_{s \in \mathcal{N}}\left[\pi_{s}\left(\left\{x_{1}, \cdots, x_{n}\right\}\right)\right]^{\omega_{s}}}{c(\Pi)} \\
& \times \mu\left(\left\{x_{1}, \cdots, x_{n}\right\}\right) d\left(x_{1}, \cdots, x_{n}\right) .
\end{aligned}
$$

Based on the GCI fusion rule, the GCI fusion for $\Pi$ is

$$
\pi_{\omega}(X)=\prod_{s \in \mathcal{N}}\left[\pi_{s}(X)\right]^{\omega_{s}} / c(\Pi)
$$

Hence, 29. can be further represented as

$$
\begin{aligned}
G(\boldsymbol{\Pi})= & -\log c(\Pi)-\log \sum_{n=0}^{\infty} \frac{1}{n !} \int \pi_{\omega}\left(\left\{x_{1}, \cdots, x_{n}\right\}\right) \\
& \times \mu\left(\left\{x_{1}, \cdots, x_{n}\right\}\right) d\left(x_{1}, \cdots, x_{n}\right) \\
= & G(\Pi)-\log \mathrm{E}_{\pi_{\omega}}[\mu(X)] .
\end{aligned}
$$

The above result reveals that $G(\boldsymbol{\Pi})$ can be decomposed into two parts: one part is $G(\Pi)$ which reflects the discrimination information between kinematic states of different sensors; the other part is $-\log E_{\pi_{\omega}}[\mu(X)]$ with $E_{\pi_{\omega}}[\mu(X)]$ being the statistical average of the GCI coefficient of conditional multilabel distribution which reflects the discrimination information between label distributions of different sensors.

Definition 3. We define the "label inconsistency indicator" with respect to a set of labeled multi-object densities $\Pi$ as

$$
d_{G}(\boldsymbol{\Pi}) \triangleq G(\boldsymbol{\Pi})-G(\Pi)=-\log E_{\pi_{\omega}}[\mu(X)] .
$$

The label inconsistency indicator $d_{G}(\boldsymbol{\Pi})$ is a measure to quantify the inconsistencies of label information embedded into multiple labeled densities. A larger value of $d_{G}(\boldsymbol{\Pi})$ indicates a higher level of label inconsistencies between densities in $\boldsymbol{\Pi}$. Moreover, the quantity $d_{G}(\boldsymbol{\Pi})$ can reflect the difference between GCI divergences of $\Pi$ and $\Pi$. The following corollary establishes upper and lower bounds on $d_{G}(\boldsymbol{\Pi})$.

Corollary 1. The following inequalities hold,

$$
0 \leqslant d_{G} \leqslant-\log \pi_{\omega}(\emptyset)
$$

with $\pi_{\omega}$ the fused density returned by GCI fusion of $\Pi$.

Proof. By definition, $\varpi_{s}(\emptyset)=1$ is always true. Therefore,

$$
\mu(\emptyset)=\prod_{s \in \mathcal{N}}\left[\varpi_{s}(\emptyset)\right]^{\omega_{s}}=1 .
$$

For $X \neq \emptyset$, the term $\mu(X)$ denotes a GCI coefficient and is therefore, within $[0,1]$. The quantity $\mathrm{E}_{\pi}[\mu(X)]$ is given by

$$
\begin{aligned}
\mathrm{E}_{\pi_{\omega}}[\mu(X)]= & \pi_{\omega}(\emptyset) \mu(\emptyset)+\sum_{n=1}^{\infty} \frac{1}{n !} \int \pi_{\omega}\left(\left\{x_{1}, \cdots, x_{n}\right\}\right) \\
& \times \mu\left(\left\{x_{1}, \cdots, x_{n}\right\}\right) d\left(x_{1}, \cdots, x_{n}\right) .
\end{aligned}
$$

Since each $\mu(\cdot)$ term within summing integrations is less than or equal to 1 , the upper bound of $\mathrm{E}_{\pi_{\omega}}[\mu(X)]$ is given by

$$
\begin{aligned}
\mathrm{E}_{\pi_{\omega}}[\mu(X)] & \leqslant \pi_{\omega}(\emptyset)+\sum_{n=1}^{\infty} \frac{1}{n !} \int \pi_{\omega}\left(\left\{x_{1}, \cdots, x_{n}\right\}\right) d\left(x_{1}, \cdots, x_{n}\right) \\
& =\int \pi_{\omega}(X) \delta X=1 .
\end{aligned}
$$

This also establishes the lower bound of $-\log \mathrm{E}_{\pi_{\omega}}[\mu(X)]$,

$$
d_{G}=-\log \mathrm{E}_{\pi_{\omega}}[\mu(X)] \geqslant 0 .
$$

On the other hand, since $\mu(\emptyset)=1$ and each $\mu\left(\left\{x_{1}, \cdots, x_{n}\right\}\right)(n \geqslant 1)$ term is non-negative, we have:

$$
\mathrm{E}_{\pi_{\omega}}[\mu(X)] \geqslant \pi_{\omega}(\emptyset)
$$

which also establishes an upper bound on $-\log \mathrm{E}_{\pi_{\omega}}[\mu(X)]$,

$$
-\log \mathrm{E}_{\pi_{\omega}}[\mu(X)] \leqslant-\log \pi_{\omega}(\emptyset) .
$$

Together, we have:

$$
0 \leqslant d_{G}=-\log \mathrm{E}_{\pi_{\omega}}[\mu(X)] \leqslant-\log \pi_{\omega}(\emptyset)
$$

From Corollary 1, we conclude that the upper bound on the quantity $d_{G}$ depends on $\pi_{\omega}(\emptyset)$. In the limit case $\pi_{\omega}(\emptyset)=0$, upper bound can reach $+\infty$.

To further investigate how the label inconsistencies affect the fusion performance, we build up the functional relationship between $d_{G}(\boldsymbol{\Pi})$ and the so-called yes-object probability and its opposite counterpart, no-object probability in the following corollary.

Corollary 2. The yes-object probability of the labeled GCI fusion $\pi_{\omega}$ can be written in terms of the label inconsistency indicator $d_{G}(\boldsymbol{\Pi})$ and the yes-object probability of the corresponding unlabeled GCI fusion $\pi_{\omega}$ as follows

$$
P_{y}\left(\boldsymbol{\pi}_{\omega}\right)=1-e^{d_{G}(\boldsymbol{\Pi})}\left[1-P_{y}\left(\pi_{\omega}\right)\right] .
$$

Proof. According to (9), we can get the no-object probabilities after GCI fusion with $\Pi$ and GCI fusion with $\Pi$ as, respec- 


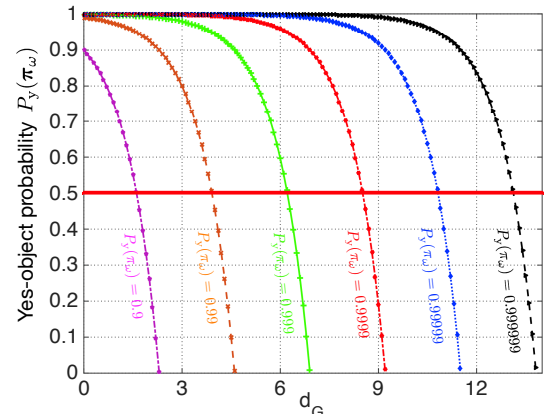

Fig. 2. Sharp decline of $P_{\mathrm{y}}\left(\boldsymbol{\pi}_{\omega}\right)$ with increasing $d_{G}(\boldsymbol{\Pi})$ for various given values of $P_{\mathrm{y}}\left(\pi_{\omega}\right)$.

tively,

$$
\begin{aligned}
P_{\mathrm{n}}\left(\boldsymbol{\pi}_{\omega}\right) & =\prod_{s \in \mathcal{N}}\left[\boldsymbol{\pi}_{s}(\emptyset)\right]^{\omega_{s}} / c(\boldsymbol{\Pi}) \\
P_{\mathrm{n}}\left(\pi_{\omega}\right) & =\prod_{s \in \mathcal{N}}\left[\pi_{s}(\emptyset)\right]^{\omega_{s}} / c(\Pi) .
\end{aligned}
$$

As for each labeled density $\boldsymbol{\pi}_{s}$ and its corresponding unlabeled version $\pi_{s}, \boldsymbol{\pi}_{s}(\emptyset)=\pi_{s}(\emptyset)$, we have

$$
P_{\mathrm{n}}\left(\boldsymbol{\pi}_{\omega}\right)=\frac{c(\Pi)}{c(\boldsymbol{\Pi})} P_{\mathrm{n}}\left(\pi_{\omega}\right)=e^{G(\boldsymbol{\Pi})-G(\Pi)} P_{\mathrm{n}}\left(\pi_{\omega}\right) .
$$

Hence, we can get the following yes-object probability

$$
P_{\mathrm{y}}\left(\boldsymbol{\pi}_{\omega}\right)=1-P_{\mathrm{n}}\left(\boldsymbol{\pi}_{\omega}\right)=1-e^{d_{G}}\left[1-P_{\mathrm{y}}\left(\pi_{\omega}\right)\right] .
$$

The above result shows that given $P_{\mathrm{y}}\left(\pi_{\omega}\right), P_{\mathrm{y}}\left(\boldsymbol{\pi}_{\omega}\right)$ monotonically decreases with $d_{G}$. As shown in Fig. 2, there can be a sharp decline for $P_{\mathrm{y}}\left(\boldsymbol{\pi}_{\omega}\right)$ if $P_{\mathrm{y}}\left(\pi_{\omega}\right)$ is close to one. Particularly, when

$$
d_{G}>-\log \pi_{\omega}(\emptyset)+\log (1-\tau),
$$

we have

$$
P_{\mathrm{y}}\left(\boldsymbol{\pi}_{\omega}\right)<\tau,
$$

and as a result, existence of no object can be inferred from GCI fusion with $\Pi$. Hence, when the label inconsistency indicator $d_{G}(\boldsymbol{\Pi})$ is too large, the GCI fusion with labeled densities can lose all tracks. Indeed, from Fig. 2, we observe that with the unlabeled yes-object probability is $P_{\mathrm{y}}\left(\pi_{\omega}\right)=0.999$, if $d_{G}$ is larger than 6.2, GCI fusion of labeled multi-object posteriors in $\Pi$ will (mis)lead us to the no-object inference since the yes-object probability $P_{\mathrm{y}}\left(\boldsymbol{\pi}_{\omega}\right)$ will be less than 0.5 .

Remark 2. Having $P_{y}\left(\boldsymbol{\pi}_{\omega}\right)<\tau$ is a sufficient condition for no-object inference, but a necessary one. A filter can also lead to an empty set estimate (no-object inference) if $P_{n}\left(\boldsymbol{\pi}_{\omega}\right)=\boldsymbol{\pi}_{\omega}(\emptyset)$ becomes larger than the probabilities of any other cardinalities $(n \neq 0)$.

\section{Summary and Motivation}

The analysis presented in this section shows that the label inconsistency indicator $d_{G}(\boldsymbol{\Pi})$ can reflect the impact of disparities between label information in different sensor nodes on the performance of GCI fusion with labeled densities. Given the labeled posteriors $\Pi$ and their unlabeled versions $\Pi$, a larger $d_{G}(\boldsymbol{\Pi})$ means

- a larger GCI divergence for $\Pi$, and thus, less optimality of GCI fusion (as it will violate PMDI more); and

- a smaller yes-object probability for the fused labeled density.

This shows that GCI fusion with labeled densities is highly sensitive to the label inconsistency indicator $d_{G}(\boldsymbol{\Pi})$ and has little tolerance (if not none) to inconsistencies between label information embedded in different local sensor nodes.

In practice, even if all the local sensors work well, the label inconsistency indicator $d_{G}(\boldsymbol{\Pi})$ can be still large. This can happen with any of the common phenomena listed in previous section, i.e., when we have an adaptive birth process in place within each local filter, or due to uncertainties in observations (excessive noise, false alarm rate, low probability of detection), or due to local pruning operations.

Revisiting example 1, we calculate the divergences $G(\boldsymbol{\Pi})$, $G(\Pi)$, the label inconsistency indicator $d_{G}(\boldsymbol{\Pi})$ and its the upper bound $-\log \pi_{\omega}(\emptyset)$. Fig. 33 a) shows those values plotted versus time. It can be seen that while GCI fusion of the unlabeled densities consistently returns small GCI divergence values, those values returned by the GCI fusion of labeled densities are large to the extent that the label inconsistency indicator $d_{G}(\boldsymbol{\Pi})$ is very close to its upper bound. This provides an in-depth explanation for the reason why GCI fusion of the LMB densities fails in this case. We also compute yes-object probabilities returned by each local filter, by GCI fusion of labeled posteriors, and by GCI fusion of unlabeled posteriors. The results are plotted in Fig. 3 (b) showing that while the two local filters and GCI fusion of unlabeled posteriors work well (in terms of successfully returning one object state estimate), GCI fusion of labeled posteriors fails.

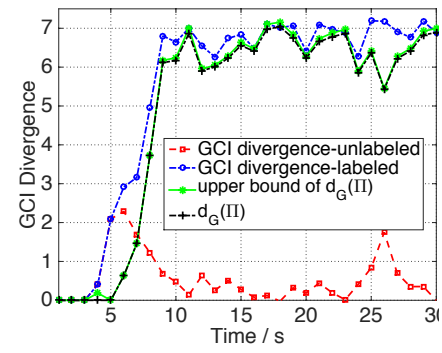

(a)

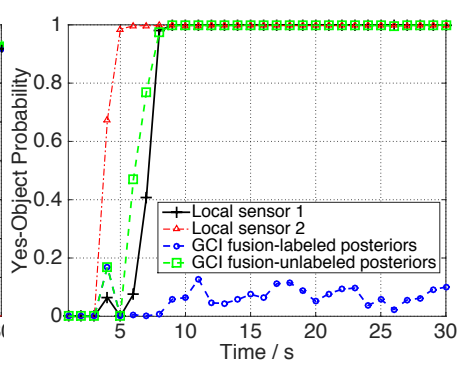

(b)
Fig. 3. (a) GCI divergences vs time; (b) Yes-object probabilities vs time.

\section{Robust Distributed Fusion: Proposed Method}

The discussions and analyses presented in previous sections show that the performance of GCI fusion with labeled densities is highly sensitive to disparities in local label information embedded in labeled posteriors at each sensor node, which is quantified by the label inconsistency indicator $d_{G}(\boldsymbol{\Pi})$. Moreover, in Section IV, the functional relationships between the GCI fusion with $\Pi$ and $\Pi$ are also founded in terms of 
the GCI divergence and the yes-object probability. Revisiting Proposition 1 and Corollary 2, we can easily conclude that

$$
\begin{gathered}
G(\Pi) \leqslant G(\boldsymbol{\Pi}) \\
P_{\mathrm{y}}\left(\pi_{\omega}\right) \geqslant P_{\mathrm{y}}\left(\boldsymbol{\pi}_{\omega}\right)
\end{gathered}
$$

which mean that GCI fusion with unlabeled posteriors $\Pi$ is expected to achieve a smaller minimal AIG value and a larger yes-object probability than GCI fusion with labeled posteriors

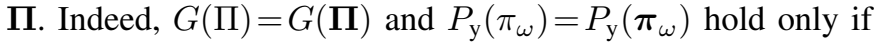

$$
\forall X \in \mathcal{F}(\mathbb{X}), \quad \mu(X)=1
$$

and this occurs only if there is $100 \%$ consistency between label-object relationships inferred from local sensor node posteriors; a condition that is hardly satisfied in practice. Hence, GCI fusion is generally expected to perform better on unlabeled posteriors assembled in $\Pi$ rather than the labeled posteriors in $\Pi$. Quality of performance of GCI fusion algorithms with unlabeled random set densities, has been already verified in several works involving particular classes of RFSs such as Poisson, i.i.d clusters and MB RFSs [6], [7], [19].

As for the task of producing tracks (equivalent to get $\left.\varpi_{\omega}(\cdot)\right)$, the fusion with the conditional multi-label distribution $\varpi_{s}(\cdot), s \in \mathcal{N}$ does not make sense when the discrimination between the statistics of labels at different sensors is large. Hence, our strategy is that if the fusion is performed at sensor $s_{0}$, the conditional multi-label distribution for the fused density $\varpi_{\omega}(\cdot)$ is produced by performing the GCI fusion with $\varpi_{s_{0}}(\cdot)$ with uniform distributions based on (9), i.e.

$$
\begin{aligned}
& \varpi_{\omega}\left(\left\{\left(\ell_{1} \mid x_{1}\right), \cdots,\left(\ell_{n} \mid x_{n}\right)\right\}\right)= \\
& \frac{\left[\varpi_{s_{0}}\left(\left\{\left(\ell_{1} \mid x_{1}\right), \cdots,\left(\ell_{n} \mid x_{n}\right)\right\}\right)\right]^{\omega_{s_{0}}}}{\sum_{\left(\ell_{1}, \cdots, \ell_{n}\right) \in \mathbb{L}_{s_{0}}^{n}}\left[\varpi_{s_{0}}\left(\left\{\left(\ell_{1} \mid x_{1}\right), \cdots,\left(\ell_{n} \mid x_{n}\right)\right\}\right)\right]^{\omega_{s_{0}}}}
\end{aligned}
$$

where $\mathbb{L}_{s_{0}}$ is the label space at sensor $s_{0}$.

The proposed robust solution to the distributed fusion of labeled posteriors is summerized as follows. Suppose that at the current time step, sensor $s_{0}$ has received agent densities from its neighbours,

Step 1: All agent labeled posteriors $\boldsymbol{\pi}_{s}(\mathbf{X})$ are marginalized to their unlabeled versions $\pi_{s}(X)$;

Step 2: Get the GCI fusion $\pi_{\omega}(X)$ with the unlabeled posteriors $\pi_{s}(X)$ based on (9);

Step 3: The labeled fused density is produced by augmenting $\pi_{\omega}(X)$ with the labels in $\mathbb{L}_{s_{0}}$, i.e.

$$
\begin{aligned}
& \boldsymbol{\pi}_{\omega}\left(\left\{\left(x_{1}, \ell_{1}\right), \cdots,\left(x_{n}, \ell_{n}\right)\right\}\right)= \\
& \varpi_{\omega}\left(\left\{\left(\ell_{1} \mid x_{1}\right), \cdots,\left(\ell_{n} \mid x_{n}\right)\right\}\right) \pi_{\omega}\left(\left\{x_{1}, \cdots, x_{n}\right\}\right)
\end{aligned}
$$

where $\varpi_{\omega}(\cdot)$ is given in 41 .

Remark 3. That the label spaces of the algorithms at different sensors are the same, i.e. $\mathbb{L}_{1}=\cdots=\mathbb{L}_{N_{s}}=\mathbb{L}$, is an inherent assumption for GCI fusion with labeled densities. However, in the practice, the real used label spaces for different sensors may be different, for example when the filters use pruning and merging strategies, or if the sensors use the adaptive birth process. Note that the proposed solution can work even if the real used label spaces are not the same, because the fusion is performed on the state space $\mathbb{X}$.

\section{A. The Unlabeled Version of Labeled RFS}

According to the proposed robust distributed fusion solution, the first step is to compute the marginals of local labeled densities on kinematic space $\mathbb{X}$. In this subsection, we investigate the unlabeled versions of common labeled densities, i.e. GLMB densities and its subclass. Firstly, we present a class of RFS defined on unlabeled state space, named as generalized multi-Bernoulli (GMB) RFS. Then we provide the mathematic representations of the unlabeled version of GLMB densities and its subclass, which turned out to be the same unlabeled RFS family, namely, GMB RFS family.

1) GMB RFS: The GMB RFS was first proposed in [19], and this paper provides a formal definition of GMB RFS.

Definition 4. A GMB RFS is an RFS on state space $\mathbb{X}$ distributed according to

$$
\pi\left(\left\{x_{1}, \cdots, x_{n}\right\}\right)=\sum_{\sigma(\mathcal{I}, \phi)} \sum_{\mathcal{F}_{n}(\mathbb{I}) \times \Phi} w^{(\mathcal{I}, \phi)} \prod_{i=1}^{n} p^{(\phi), \mathcal{I}^{v}(i)}\left(x_{\sigma(i)}\right)
$$

where $\sigma$ denotes one permutation of $\mathcal{I}, \sigma(i)$ denotes the ith element of the permutation, the summation $\sum_{\sigma}$ is taken over all permutations on the numbers $1, \cdots, n, \Phi$ is a discrete space, $\mathbb{I}$ is the set of indexes of densities, $\mathcal{I}^{v} \in \mathbb{I}^{|\mathcal{I}|}$ is a vector constructed by sorting the elements of the set $\mathcal{I}, w^{(\mathcal{I}, \phi)}$ and $p^{(\phi), \imath}(x)$ satisfy

$$
\begin{aligned}
\sum_{\mathcal{I} \in \mathcal{F}(\mathbb{I})} \sum_{\phi \in \Phi} w^{(\mathcal{I}, \phi)} & =1, \\
\int p^{(\phi), \imath}(x) d x & =1, \imath \in \mathbb{I} .
\end{aligned}
$$

A GMB distribution is constructed by a set of hypotheses, $\{(\mathcal{I}, \phi):(\mathcal{I}, \phi) \in \mathcal{F}(\mathbb{I}) \times \Phi\}$. We define a set of densities for each $\phi \in \Phi$ as:

$$
\mathfrak{P}^{(\phi)} \triangleq\left\{p^{(\phi), \imath}(x)\right\}_{\imath \in \mathbb{I}} .
$$

Under each hypothesis, the corresponding weight is $w^{(\mathcal{I}, \phi)}$ and the corresponding density set is $\mathfrak{P}^{(\phi)}$. Thus a GMB density is completely characterized by the set of parameters $\left\{\left(w^{(\mathcal{I}, \phi)}, \mathfrak{P}^{(\phi)}\right):(\mathcal{I}, \phi) \in \mathcal{F}(\mathbb{I}) \times \Phi\right\}$.

The first-order moment of a GMB RFS distributed according to 43 is given by: [19]

$$
v(x)=\sum_{\imath \in \mathbb{I}} r^{(\imath)} p^{(\imath)}(x) .
$$

where

$$
\begin{aligned}
r^{(\imath)} & =\sum_{\mathcal{I} \in \mathcal{F}(\mathbb{I})} \sum_{\phi \in \Phi} 1_{\mathcal{I}}(\imath) w^{(\mathcal{I}, \phi)}, \\
p^{(\imath)}(x) & =\sum_{\mathcal{I} \in \mathcal{F}(\mathbb{I})} \sum_{\phi \in \Phi} 1_{\mathcal{I}}(\imath) w^{(\mathcal{I}, \phi)} p^{(\phi), \imath}(x) / r^{(\imath)} .
\end{aligned}
$$

2) Unlabeled Versions of Common Labeled Densities: We present the mathematical representations of the unlabeled versions for GLMB density and its subclass, LMB density. 
Proposition 2. If a labeled RFS $\mathrm{X}$ on $\mathbb{X} \times \mathbb{L}$ is a GLMB RFS distributed according to (4), then $X=\mathcal{K}(\mathbf{X})$ is distributed as

$$
\begin{aligned}
& \pi\left(\left\{x_{1}, \cdots, x_{n}\right\}\right)= \\
& \sum_{\sigma} \sum_{(I, c) \in \mathcal{F}_{n}(\mathbb{L}) \times \mathbb{C}} w^{(I, c)} \prod_{i=1}^{n} p^{(c), I^{v}(i)}\left(x_{\sigma(i)}\right)
\end{aligned}
$$

where

$$
\begin{aligned}
w^{(I, c)} & \triangleq w^{(c)}(I), I \in \mathcal{F}(\mathbb{L}) \\
p^{(c), \ell}(x) & \triangleq p^{(c)}(x, \ell), \ell \in \mathbb{L} .
\end{aligned}
$$

Proof. See Appendix A.

Proposition 3. If a labeled RFS $\mathbf{X}$ on $\mathbb{X} \times \mathbb{L}$ is an LMB RFS distributed according to $(6)$, then $X=\mathcal{K}(\mathbf{X})$ is distributed as:

$$
\pi\left(\left\{x_{1}, \cdots, x_{n}\right\}\right)=\sum_{\sigma} \sum_{I \in \mathcal{F}_{n}(\mathbb{L})} w^{(I)} \prod_{i=1}^{n} p^{\left(I^{v}(i)\right)}\left(x_{\sigma(i)}\right)
$$

where

$$
\begin{aligned}
w^{(I)} & \triangleq w(I), I \in \mathcal{F}(\mathbb{L}) \\
p^{(\ell)}(x) & \triangleq p(x, \ell), \ell \in \mathbb{L} .
\end{aligned}
$$

Proof. See Appendix B.

Remark 4. Propositions 2 and 3 explicitly describe the relationship between the parameters of GLMB and LMB densities and the parameters of their unlabeled versions, respectively. Specifically, the unlabeled version of GLMB is a GMB with $\mathbb{I}=\mathbb{L}$ and $\Phi=\mathbb{C}$; the unlabeled version of LMB is a GMB with and $\mathbb{I}=\mathbb{L}$ and the discrete space $\Phi$ only has one point. Note that (51) is an MB density with a set of parameters $\left\{r^{(\ell)}, p^{(\ell)}(x)\right\}_{\ell \in \mathbb{L}}$ in nature.

\section{B. GCI Fusion with GMB Distributions}

Once the labeled multi-object posteriors in GLMB RFS family are marginalized to the GMB densities based on Propositions 2 3, the subsequent task is to perform GCI fusion with GMB densities according to the proposed robust solution. We present the formula of GCI fusion with two GMB densities in this subsection, and for the case of more than two densities, a common method is to perform the pair-wise fusion [7], [19]. Actually, a manipulatable formula for GCI fusion has two implicit demands: one is the formula should make the computation of the set integral in (9) tractable; the other is the fused distribution should belong to the same RFS family of local posteriors or its unlabeled version, to enable the pairwise fusion of more than two densities. In the following, we are devoted to deriving an explicit formula for GCI fusion with GMB distributions to satisfy these two demands.

The following Definition 4 first provided in [19] describes the degree of separation for different tracks from the view of highest posterior density (HPD) [36] region. With this definition, [19] also provides an approximation technique to simplify the fractional order exponential power of MB distribution. Since they are pertinent to the derivation that follows, we firstly introduce them here.

Definition 5. Consider an MB posterior $\pi=$ $\left\{\left(r^{(\imath)}, p^{(\imath)}(\cdot)\right)\right\}_{\imath \in \mathbb{I}}$, where $\mathbb{I}$ is the index set of Bernoulli components. If $\mathbb{X}_{\imath}$ is the HPD of confidence $\lambda$ for $p^{(\imath)}(\cdot)$, then the Bernoulli components of $\pi(X)$ are said to be mutually $\lambda \times 100 \%$ separated if,

$$
\forall \imath \neq \imath^{\prime}, \quad \mathbb{X}_{\imath} \cap \mathbb{X}_{\imath^{\prime}}=\emptyset
$$

The approximation technique proposed in [19] is that if the Bernoulli components of an MB posterior density denoted by $\pi=\left\{\left(r^{(\imath)}, p^{(\imath)}(\cdot)\right)\right\}_{\imath \in \mathbb{I}}$ are mutually $\lambda \times 100 \%$ separated and $\lambda$ is very close to 1 (e.g. $\lambda \geqslant 0.9$ ), then

$$
\pi\left(\left\{x_{1}, \ldots, x_{n}\right\}\right)^{\omega} \approx \sum_{\sigma} \sum_{\mathcal{I} \in \mathcal{F}_{n}(\mathbb{I})}\left[w^{(\mathcal{I})}\right]^{\omega}\left[\prod_{i=1}^{n} p^{\left(\mathcal{I}^{v}(i)\right)}\left(x_{\sigma(i)}\right)\right]^{\omega}
$$

where $w^{(\mathcal{I})}=\prod_{\imath \in \mathcal{I}} r^{(\imath)} \prod_{\imath^{\prime} \in \mathbb{I}-\mathcal{I}}\left(1-r^{\left(\imath^{\prime}\right)}\right)$.

Consider two GMB distributions $\pi_{s}, s=1,2$, which are the unlabeled versions of posteriors output by two sensors in a network, i.e.

$$
\begin{aligned}
& \pi_{s}\left(\left\{x_{1}, \cdots, x_{2}\right\}\right)= \\
& \sum_{\sigma_{s}(\mathcal{I}, \phi) \in \mathcal{F}_{n}\left(\mathbb{I}_{s}\right) \times \Phi_{s}} \omega_{s}^{(\mathcal{I}, \phi)} \prod_{i=1}^{n} p_{s}^{(\phi), \mathcal{I}^{v}(i)}\left(x_{\sigma_{s}(i)}\right), s=1,2 .
\end{aligned}
$$

According to GCI fusion rule, by substitution of (54) into (9), we are faced with a tough task that is to simplify the following expression involving the fractional order exponential power,

$$
\pi_{s}^{\omega_{s}}=\left[\sum_{\sigma} \sum_{(\mathcal{I}, \phi) \in \mathcal{F}_{n}\left(\mathbb{I}_{s}\right) \times \Phi_{s}} \omega_{s}^{(\mathcal{I}, \phi)} \prod_{i=1}^{n} p_{s}^{(\phi), \mathcal{I}^{v}(i)}\left(x_{\sigma(i)}\right)\right]^{\omega_{s}} .
$$

Obviously, (55) is computationally intractable due to the sum over the discrete space $\mathcal{F}_{n}\left(\mathbb{I}_{s}\right) \times \Phi$ and over $\sigma$.

Herein, we adopt a two-step approximation strategy that is to approximate the GMB distribution as a more tractable distribution preserving its key statistical properties firstly and then compute the GCI fusion of the approximated distributions. On one hand, in the RFS based multi-object tracking algorithms, approximating the multi-object posterior as a simple distribution preserving its key statistical properties can usually obtain a great reduction in computation burden with a slight compromise in accuracy. For instance, in LMB filter, the multi-object posterior is approximated as an LMB distribution which preserves its first-order moment, and the performance of LMB filter has been well demonstrated in [23]. On the other hand, by adopting the approximation technique in [19], the MB density can accommodate simplification of the fractional order exponential power in (55) manageable as shown in (53). Inspired by these two aspects, we seek the MB approximation which preserve the first-order moment of the original GMB distribution in this paper.

For the subsequent development, we firstly give the definition of fusion map.

Definition 6. Without loss of generality, assume that $\left|\mathbb{I}_{1}\right| \leq$ $\left|\mathbb{I}_{2}\right|$. A fusion map (for the current time) is a function $\tau: \mathbb{I}_{1} \rightarrow$ $\mathbb{I}_{2}$ such that $\tau(i)=\tau\left(i^{*}\right)$ implies $i=i^{*}$. The set of all such fusion maps is called fusion map space denoted by $\mathcal{T}$. The subset of $\mathcal{T}$ with domain $\mathcal{I}$ is denoted by $\mathcal{T}(\mathcal{I})$. For notation convenience, we define $\tau(I) \triangleq\{\tau(i), i \in I\}$. 
Remark 5. Note that each fusion map denotes a hypothesis that the tracks in sensor 2 are one-to-one matching with the tracks in sensor 1. The fusion map plays the similar role as the measurement-track association map in $\delta$-GLMB filter [24].

The two-step approximation strategy is described as follow:

$>$ The MB approximation of GMB density preserving the firstorder moment: According to (46), the first-order moments of the GMB densities of form (54) are given by

$$
v_{s}(x)=\sum_{\imath \in \mathbb{I}_{s}} \widetilde{r}_{s}^{(\imath)} \widetilde{p}_{s}^{(\imath)}(x), s=1,2
$$

where

$$
\begin{aligned}
\widetilde{r}_{s}^{(\imath)} & =\sum_{\mathcal{I} \in \mathcal{F}\left(\mathbb{I}_{s}\right)} \sum_{\phi \in \Phi_{s}} 1_{\mathcal{I}}(\imath) w_{s}^{(\mathcal{I}, \phi)} \\
\widetilde{p}_{s}^{(\imath)}(x) & =\sum_{\mathcal{I} \in \mathcal{F}\left(\mathbb{I}_{s}\right)} \sum_{\phi \in \Phi_{s}} 1_{\mathcal{I}}(\imath) w_{s}^{(\mathcal{I}, \phi)} p_{s}^{(\phi), \imath}(x) / \widetilde{r}_{s}^{(\imath)}
\end{aligned}
$$

The above expression can be interpreted as a weighted sum of the densities of all individual tracks, with each track denoted by a Bernoulli $\operatorname{RFS}\left\{\left(\widetilde{r}_{s}^{(\imath)}, \widetilde{p}_{s}^{(\imath)}\right)\right\}, \imath \in \mathbb{I}_{s}$. Hence, the MB distributions that match exactly the first-order moments of the GMB densities of form (54) are

$$
\widetilde{\pi}_{s}=\left\{\left(\widetilde{r}_{s}^{(\imath)}, \widetilde{p}_{s}^{(\imath)}(x)\right)\right\}_{\imath \in \mathbb{I}_{s}}, s=1,2 .
$$

$>$ The GCI fusion with MB approximations: By adopting the approximation technique in (53) proposed in [19], we have on condition that the Bernoulli components of $\tilde{\pi}_{s}=$ $\left\{\left(\widetilde{r}_{s}^{(\imath)}, \widetilde{p}_{s}^{(\imath)}(x)\right)\right\}_{\imath \in \mathbb{I}_{s}}$ are mutually $\lambda \times 100 \%$ separated with $\lambda$ close to 1 , the fractional order exponential power of the MB distribution can be simplified as

$\widetilde{\pi}_{s}\left(\left\{x_{1} ; \cdot, x_{n}\right\}\right)^{\omega_{s}} \approx \sum_{\sigma_{s} \mathcal{I}_{s} \in \mathcal{F}_{n}\left(\mathbb{I}_{s}\right)}\left[\widetilde{w}_{s}^{\left(\mathcal{I}_{s}\right)}\right]^{\omega_{s}}\left[\prod_{i=1}^{n} \widetilde{p}_{s}^{\left(\mathcal{I}_{s}^{v}(i)\right)}\left(x_{i}\right)\right]^{\omega_{s}}$

where $\widetilde{w}_{s}^{\left(\mathcal{I}_{s}\right)}=\prod_{\imath \in \mathcal{I}_{s}} r_{s}^{(\imath)} \prod_{\imath^{\prime} \in \mathbb{I}_{s}-\mathcal{I}_{s}}\left(1-\widetilde{r}_{s}^{(\imath)}\right)$. By substitution of $(60)$ for $s=1,2$ into $(9)$, and utilizing Definition 5, according to Proposition 3 given in [19], the fused density can be computed as

$$
\begin{aligned}
& \pi_{\omega}\left(\left\{x_{1}, \ldots, x_{n}\right\}\right) \\
= & \frac{1}{C} \prod_{s=1,2} \sum_{\sigma_{s}} \sum_{\mathcal{I}_{s} \in \mathcal{F}_{n}\left(\mathbb{I}_{s}\right)}\left[\widetilde{w}_{s}^{\left(\mathcal{I}_{s}\right)}\right]^{\omega_{s}}\left[\prod_{i=1}^{n} \widetilde{p}_{s}^{\left(\mathcal{I}_{s}^{v}(i)\right)}\left(x_{i}\right)\right]^{\omega_{s}} \\
= & \sum_{\sigma} \sum_{(\mathcal{I}, \tau) \in \mathcal{F}_{n}\left(\mathbb{I}_{1}\right) \times \mathcal{T}(\mathcal{I})} w_{\omega}^{(\mathcal{I}, \tau)} \prod_{i=1}^{n} p_{\omega}^{(\tau), \mathcal{I}^{v}(i)}\left(x_{\sigma(i)}\right)
\end{aligned}
$$

where

$$
\begin{aligned}
w_{\omega}^{(\mathcal{I}, \tau)} & =\check{w}_{\omega}^{(\mathcal{I}, \tau)} / C \\
p_{\omega}^{(\tau), \imath}(x) & =\widetilde{p}_{\omega}^{(\imath, \tau(\tau))}(x) \\
\eta_{\omega}^{(\tau), \imath} & =\widetilde{\eta}_{\omega}^{(\imath, \tau(\imath))} \\
\check{w}_{\omega}^{(\mathcal{I}, \tau)} & =\left[\widetilde{w}_{1}^{(\mathcal{I})}\right]^{\omega_{1}}\left[\widetilde{w}_{2}^{(\tau(\mathcal{I}))}\right]^{\omega_{2}} \prod_{\imath \in \mathcal{I}} \eta_{\omega}^{(\tau), \imath} \\
C & =\int \pi_{\omega}(X) \delta X=\sum_{(\mathcal{I}, \tau) \in \mathcal{F}\left(\mathbb{I}_{1}\right) \times \mathcal{T}(\mathcal{I})} \check{w}^{(\mathcal{I}, \tau)} .
\end{aligned}
$$

with

$$
\begin{aligned}
\widetilde{p}_{\omega}^{(\imath, \jmath)}(x) & =\left[\widetilde{p}_{1}^{(\imath)}(x)\right]^{\omega_{1}}\left[\widetilde{p}_{2}^{(\jmath)}(x)\right]^{\omega_{2}} / \widetilde{\eta}_{\omega}^{(\imath, \jmath)},(\imath, \jmath) \in \mathbb{I}_{1} \times \mathbb{I}_{2} \\
\widetilde{\eta}_{\omega}^{(\imath, \jmath)} & =\int\left[\widetilde{p}_{1}^{(\imath)}(x)\right]^{\omega_{1}}\left[\widetilde{p}_{2}^{(\jmath)}(x)\right]^{\omega_{2}} d x,(\imath, \jmath) \in \mathbb{I}_{1} \times \mathbb{I}_{2}
\end{aligned}
$$

Finally, via the aforementioned two-step approximation, we derive the explicit formula for GCI fusion with two GMB distributions as shown in 61). It can be observed that 61) is another GMB distribution, it can enable the distributed fusion with GLMB RFS family in a sensor network owning more than two sensors by applying pair-wise fusion.

Remark 6. Usually the true single object state corresponding to each Bernoulli component $\left\{\left(\widetilde{r}_{s}^{(l)}, \widetilde{p}_{s}^{(l)}\right)\right\}$ determines the center of its HPD region. Furthermore, the width of HPD region of a Bernoulli component is smaller with lower maneuverability and higher SNR. In such practical scenarios, the MB approximation preserving the first order moment of the original GMB posterior can be easily assumed to be mutually separated with very high confidence, and the condition of the approximation in 60) can be easily satisfied.

\section{Construction of the Labeled Fused Posterior}

After fusing the unlabeled densities and obtained the GMB fused density $\pi_{\omega}$, one can extract the multiple object states directly from the fused GMB density if the trajectories are not required; otherwise if the system does need distinguish object identities and form the trajectories, we can also construct the labeled density based on (41) and (42).

The conditional multi-label distribution $\varpi_{s_{0}}(\cdot)$ in 41) can be computed from $\pi_{s_{0}}(\mathbf{X})$, i.e., the local labeled density of sensor $s_{0}$, by combination of (17) and (18), however, it is hard to obtain an analytic solution even for GLMB densities. As a result, the calculations of (41) and (42) are even more difficult. However, we find that if $\varpi_{s_{0}}(\cdot)$ is replaced by $\bar{\varpi}_{s_{0}}(\cdot)$ of the first-order moment preserved LMB approximation of $\boldsymbol{\pi}_{s_{0}}(\mathbf{X})$, the calculation of 42 can be dramatically simplified, and more importantly, the labeled fused density is another GLMB.

Assume that the labeled posterior $\boldsymbol{\pi}_{s_{0}}(\mathbf{X})$ is a GLMB density of form (4), i.e.,

$$
\boldsymbol{\pi}_{s_{0}}(\mathbf{X})=\Delta(\mathbf{X}) \sum_{c \in \mathbb{C}_{s_{0}}} w_{s_{0}}^{(c)}(\mathcal{L}(\mathbf{X}))\left[p_{s_{0}}^{(c)}\right]^{\mathbf{X}}
$$

According to Proposition 4 in [24], the LMB approximation which preserves the first-order moment of $\boldsymbol{\pi}_{s_{0}}(\mathbf{X})$ is

$$
\overline{\boldsymbol{\pi}}_{s_{0}}(\mathbf{X})=\left\{\left(\bar{r}_{s_{0}}^{(\ell)}, \bar{p}_{s_{0}}^{(\ell)}(x)\right)\right\}_{\ell \in \mathbb{L}_{s_{0}}}
$$

with

$$
\bar{r}_{s_{0}}^{(\ell)}=\sum_{I \in \mathcal{F}\left(\mathbb{L}_{s_{0}}\right)} \sum_{c \in \mathbb{C}_{s_{0}}} 1_{I}(\ell) w_{s_{0}}^{(c)}(I),
$$

$\bar{p}_{s_{0}}^{(\ell)}(x)=\sum_{I \in \mathcal{F}\left(\mathbb{L}_{s_{0}}\right)} \sum_{c \in \mathbb{C}_{s_{0}}} 1_{I}(\ell) w_{s_{0}}^{(c)}(I) p_{s_{0}}^{(c)}(x, \ell) / \bar{r}_{s_{0}}^{(\ell)}$

According to (17) and (18), the conditional joint probability density of $\ell_{1}, \cdots, \ell_{n}$ of $\overline{\boldsymbol{\pi}}_{s_{0}}(\mathbf{X})$ can be computed by

$$
\begin{aligned}
& \bar{\varpi}_{s_{0}}\left(\left\{\left(\ell_{1} \mid x_{1}\right), \cdots,\left(\ell_{n} \mid x_{n}\right)\right\}\right)= \\
& \frac{\bar{w}_{s_{0}}\left(\left\{\ell_{1}, \cdots, \ell_{n}\right\}\right) \prod_{i=1}^{n} \bar{p}_{s_{0}}^{\left(\ell_{i}\right)}\left(x_{i}\right)}{\sum_{\left(\ell_{1}, \cdots, \ell_{n}\right) \in \mathbb{L}_{s_{0}}^{n}} \bar{w}_{s_{0}}\left(\left\{\ell_{1}, \cdots, \ell_{n}\right\}\right) \prod_{i=1}^{n} \bar{p}_{s_{0}}^{\left(\ell_{i}\right)}\left(x_{i}\right)}
\end{aligned}
$$


where $\bar{w}_{s_{0}}(I)=\prod_{\ell \in I} \bar{r}_{s_{0}}^{(\ell)} \prod_{\ell^{\prime} \in \mathbb{L}_{s}-I}\left(1-\bar{r}_{s_{0}}^{\left(\ell^{\prime}\right)}\right)$. Replacing $\varpi_{s_{0}}(\cdot)$ with $\bar{\varpi}_{s_{0}}(\cdot)$, according to (41), by simple deduction we can obtain the corresponding $\bar{\varpi}_{\omega}(\cdot)$ as

$$
\begin{aligned}
& \bar{\varpi}_{\omega}\left(\left\{\left(\ell_{1} \mid x_{1}\right), \cdots,\left(\ell_{n} \mid x_{n}\right)\right\}\right)= \\
& \frac{\left[\bar{w}_{s_{0}}\left(\left\{\ell_{1}, \cdots, \ell_{n}\right\}\right)\right]^{\omega_{s_{0}}} \prod_{i=1}^{n}\left[\bar{p}_{s_{0}}^{\left(\ell_{i}\right)}\left(x_{i}\right)\right]^{\omega_{s_{0}}}}{\sum_{\left(\ell_{1}, \cdots, \ell_{n}\right) \in \mathbb{L}_{s_{0}}^{n}}\left[\bar{w}_{s_{0}}\left(\left\{\ell_{1}, \cdots, \ell_{n}\right\}\right)\right]^{\omega_{s_{0}}} \prod_{i=1}^{n}\left[\bar{p}_{s_{0}}^{\left(\ell_{i}\right)}\left(x_{i}\right)\right]^{\omega_{s_{0}}}} .
\end{aligned}
$$

Without loss of generalisation, let $s_{0}=1$, we can obtain the following proposition:

Proposition 4. If the labeled fused density is constructed as

$$
\begin{aligned}
& \boldsymbol{\pi}_{\omega}\left(\left\{\left(x_{1}, \ell_{1}\right), \cdots,\left(x_{n}, \ell_{n}\right)\right\}\right)= \\
& \quad \bar{\varpi}_{\omega}\left(\left\{\left(\ell_{1} \mid x_{1}\right), \cdots,\left(\ell_{n} \mid x_{n}\right)\right\}\right) \pi_{\omega}\left(\left\{x_{1}, \cdots, x_{n}\right\}\right)
\end{aligned}
$$

where $\pi_{\omega}(\cdot)$ and $\bar{\varpi}_{w}(\cdot)$ is given in (61) and (74) (with $s_{0}=1$ ) respectively, then the labeled fused density is given by

$$
\boldsymbol{\pi}_{\omega}(\mathbf{X})=\Delta(\mathbf{X}) \sum_{\tau \in \mathcal{T}} 1_{\mathcal{T}(\mathcal{L}(\mathbf{X}))}(\tau) w_{\omega}^{(\tau)}(\mathcal{L}(\mathbf{X}))\left[p_{\omega}^{(\tau)}\right]^{\mathbf{X}}
$$

defined on $\mathbb{L}_{\omega}=\mathbb{I}_{1}=\mathbb{L}_{1}$, where

$$
\begin{aligned}
p_{\omega}^{(\tau)}(\cdot, \ell) & \triangleq p_{\omega}^{(\tau), \ell}(\cdot) \\
w_{\omega}^{(\tau)}(I) & \triangleq w_{\omega}^{(I, \tau)} .
\end{aligned}
$$

Proof. See Appendix C.

Remark 7. Note that constructing the labeled fused density in (76) from the fused GMB density in (61) does not require any additional computation because (76) is completely determined by the parameters of fused GMB density according to Proposition 4. Moreover, it is obvious that the constructed GLMB fused density has the same cardinality distribution and the (unlabeled) first-order moment as the fused GMB density.

\section{Summary and Discussions}

In this section, we proposed a novel distributed fusion solution for label densities. More specifically, we proposed a robust GCI fusion algorithms for GLMB densities (R-GCIGLMB) according to the proposed solution. The schematic of the R-GCI-GLMB fusion algorithm is shown in Fig. 4. One should note that R-GCI-GLMB fusion algorithm also suitable for the fusion with LMB filters or $\mathrm{M} \delta$-GLMB filters. For the LMB filter, approximating GMB density as a first-order moment preserved $\mathrm{MB}$ density is not required, because the unlabeled version of an LMB posterior is just an MB density. After the fused GLMB density is constructed, if feedback is required, the fused GLMB density should be approximated as the same class of local posterior, such as the LMB or $\mathrm{M} \delta$ GLMB densities.

In the following, we provide the pseudocode of the $\mathrm{R}$ GCI-GLMB fusion algorithm in three parts (algorithms). Algorithm 1 shows the pseudocode for approximating a GMB density as an MB density matching the first-order moment. Algorithm 2 shows the pseudocode of GCI fusion with GMB densities from $N_{s}\left(N_{s} \geqslant 2\right)$ sensors, where the pair-wise fusion strategy is adopted and the ordering of pair-wise fusions is irrelevant. Taking the case that local sensors perform GLMB

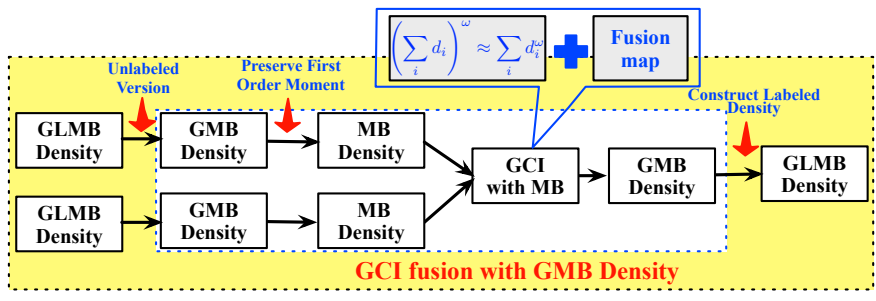

Fig. 4. A schematic diagram of the R-GCI-GLMB fusion algorithm.

filtering as an example, Algorithm 3 shows the pseudocode of the whole fusion algorithm.

The computational cost of the R-GCI-GLMB algorithm mainly lies in the GCI fusion of GMB densities, since marginalizing the GLMB density to its unlabelled version and constructing the fused GLMB density from the fused GMB density are only conceptual operations with no need to calculate any quantities. Taking the Gaussian mixture (GM) implementation as an example, we analyse the computational complexity of the R-GCI-GLMB fusion algorithm by the following comparison of the GCI fusion with the CPHD filter (GCI-CPHD) proposed in [6], [7]. Suppose that each singleobject density for the local GLMB filter is approximated by $M_{G}$ Gaussian components, and the location density of each local CPHD filter is approximated by $n_{\max } M_{G}$ Gaussian components, where $n_{\max }$ denotes the maximum number of objects. The computational cost of the R-GCI-GLMB fusion mainly depends on two parts: one is for computing the fused singleobject densities $\widetilde{p}_{\omega}^{(\imath, j)}$ which has the computational complexity $\mathcal{O}\left[\left(\left|\mathbb{I}_{\max }\right| M_{G}\right)^{N_{s}}\right]$, where $\mathbb{I}_{\max }=\arg \max _{\mathbb{I} \in\left\{\mathbb{I}_{s}: s \in \mathcal{N}\right\}}|\mathbb{I}| ;$ the other part is for the calculation of the fused weights $w_{\omega}^{(\mathcal{I}, \tau)}$ which has the computational complexity $\mathcal{O}\left[N_{s} N_{H}\right]$ where $N_{H}=\left|\mathcal{F}\left(\mathbb{I}_{\text {max }}\right) \times \mathcal{T}_{\text {max }}\right|$ with $\mathcal{T}_{\text {max }}$ being the fusion map space defined on $\mathbb{I}_{\max }$. Overall, the R-GCI-GLMB fusion has the computational complexity $\mathcal{O}\left[\max \left\{N_{s} N_{H},\left(\left|\mathbb{I}_{\max }\right| M_{G}\right)^{N_{s}}\right\}\right]$. By contrast, the computational complexity of the GCI-CPHD fusion is $\mathcal{O}\left[\left(n_{\max } M_{G}\right)^{N_{s}}\right]$. As it is common with the GM implementation of stochastic filters, the fused Gaussian components with negligible coefficients are pruned to reduce the computational cost when implementing the GCI-CPHD fusion [7]. When implementing the R-GCI-GLMB fusion, we apply the same strategy to reduce the computational cost of the first part. Moreover, to keep the computational cost of the second part moderate, some common efficient strategies, such as the truncation of the fused GMB density with the ranked assignment strategy [22], [23], are adopted.

\section{Performance Assessment}

In this section, the performance of the proposed R-GCIGLMB fusion is examined by comparison with the state of art over distributed sensor networks. The GM implementations are adopted for all the distributed tracking algorithms.

The standard object and observation models [23] are used. The object state variable is a vector of planar position and velocity $x_{k}=\left[\begin{array}{llll}p_{x, k} & p_{y, k} & \dot{p}_{x, k} & \dot{p}_{y, k}\end{array}\right]^{\top}$, where "T" denotes 

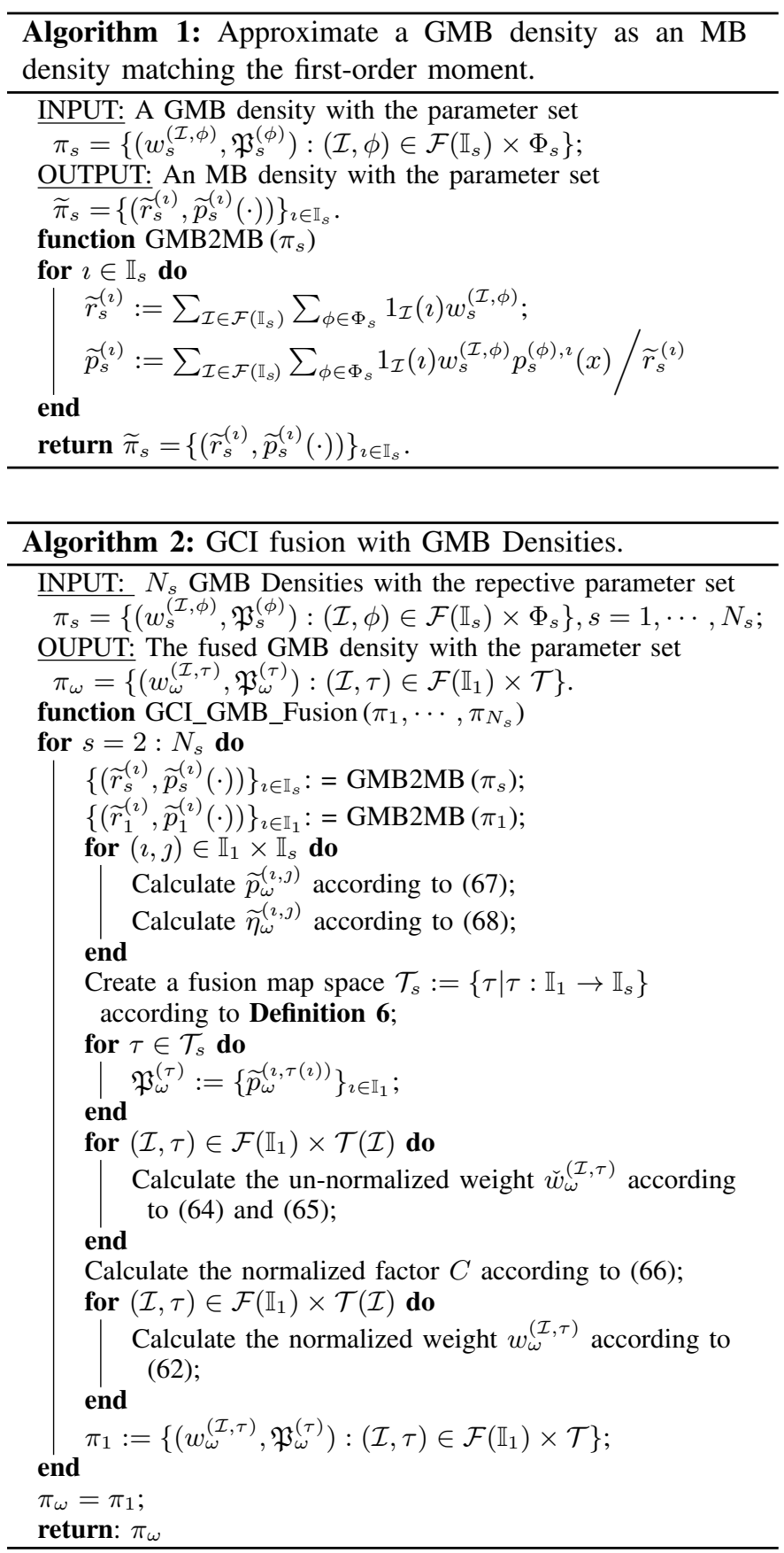

matrix transpose. The single-object transition model is the linear Gaussian

$$
f_{k}\left(x_{k} \mid x_{k-1}\right)=\mathcal{N}\left(x_{k} ; \mathbf{F}_{k} x_{k-1}, \mathbf{Q}_{k}\right)
$$

with its parameters given for a nearly constant velocity model:

$$
\mathbf{F}_{k}=\left[\begin{array}{cc}
\mathbf{I}_{2} & \Delta \mathbf{I}_{2} \\
\mathbf{0}_{2} & \mathbf{I}_{2}
\end{array}\right], \quad \mathbf{Q}_{k}=\sigma_{v}^{2}\left[\begin{array}{cc}
\frac{1}{4} \mathbf{I}_{2} & \frac{1}{2} \Delta \mathbf{I}_{2} \\
\frac{1}{3} \mathbf{0}_{2} & \mathbf{I}_{2}
\end{array}\right]
$$

where $\mathbf{I}_{2}$ and $\mathbf{0}_{2}$ denote the $2 \times 2$ identity and zero matrices, $\Delta=1 \mathrm{~s}$ is the sampling period, and $\sigma_{\nu}=5 \mathrm{~m} / \mathrm{s}^{2}$ is the standard deviation of the process noise. The state independent survival probability of the object is given by $P_{S, k}=0.98$.

Two types of birth procedures are considered in different experiments. One is the prior knowledge-based birth procedure.

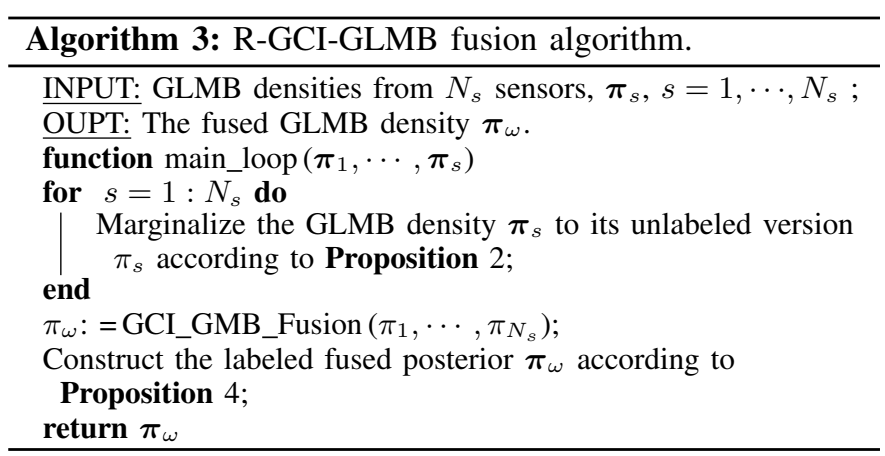

At each time $k$, the birth process is an LMB RFS with the parameter set $\boldsymbol{\pi}_{B}=\left\{\left(r_{B}^{(k, i)}, p_{B}^{(k, i)}\right)\right\}_{i=1}^{3}$ where $r_{B}^{(k, i)}=0.04$ and $p_{B}^{(k, i)}=\mathcal{N}\left(x ; m_{B}^{(i)}, P_{B}\right)$ with $m_{B}^{(1)}=\left[\begin{array}{lllll}200 & 400 & 0 & 0\end{array}\right]^{\top}$,

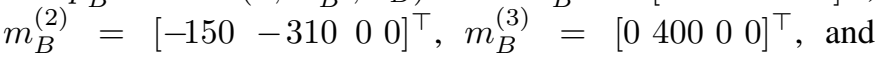
$P_{B}=\operatorname{diag}\left(\left[\begin{array}{llll}900 & 900 & 400 & 400\end{array}\right]\right)$.

The other birth procedure is the adaptive birth procedure proposed in [24]. The LMB birth process at time step $k+1$ depends on the measurement set $Z$ of the current time step $k$ and is given by

$$
\boldsymbol{\pi}_{B}=\left\{r_{B}^{(k+1, i)}(z), p_{B}^{(k+1, i)}(x \mid z)\right\}_{z \in Z} .
$$

More specifically, the existence probability $r_{B}^{(k+1, i)}(z)$ is proportional to the probability that $z$ is not assigned to any track during the update at time step $k$ :

$$
r_{B}^{(k+1, i)}(z)=\min \left(r_{B, \max }, \frac{1-r_{U, k}(z)}{\sum_{\xi \in Z} 1-r_{U, k}(\xi)} \cdot \lambda_{B, k+1}\right)
$$

where $r_{U, k}(z)$ denotes the probability that a measurement $z$ is associated to a track in the hypotheses at time step $k$, $\lambda_{B, k+1}$ is the expected number of object births at time step $k+1$, and $r_{B, \max } \in[0,1]$ is the maximum existence probability of a newly born object. Each density $p_{B}^{(k+1, i)}(x \mid z)=$ $\mathcal{N}\left(x ; m_{B}(z), P_{B}\right)$ with $m_{B}(z)=\left[\begin{array}{llll}z(1) & z(2) & 0 & 0\end{array}\right]^{\top}, P_{B}=$

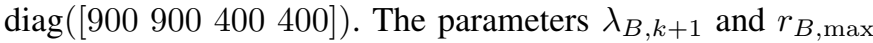
are set to be 0.8 and 0.3 , respectively. The details about how to compute the probability $r_{U, k}(z)$ are given in [24].

Each sensor node detects an object independently with the same probability $P_{D, k}$. The single-object observation model is linear Gaussian

$$
g_{k}\left(z \mid \mathbf{x}_{k}\right)=\mathcal{N}\left(z ; \mathbf{H}_{k} \mathbf{x}_{k}, \mathbf{R}_{k}\right)
$$

with parameters

$$
\mathbf{H}_{k}=\left[\begin{array}{ll}
\mathbf{I}_{2} & \mathbf{0}_{2}
\end{array}\right], \quad \mathbf{R}_{k}=\sigma_{\varepsilon}^{2} \mathbf{I}_{2}
$$

where $\sigma_{\varepsilon}=25 \mathrm{~m}$ is the standard deviation of the measurement noise. The number of clutter reports in each scan is Poisson distributed with $\lambda=10$. Each clutter report is sampled uniformly over the whole surveillance region.

The optimal sub-pattern assignment (OSPA) error [37] serves as the main performance metric with the cut-off value $c=100 \mathrm{~m}$ and the order parameter $p=1$. All performance metrics are averaged over 200 Monte Carlo (MC) runs. 


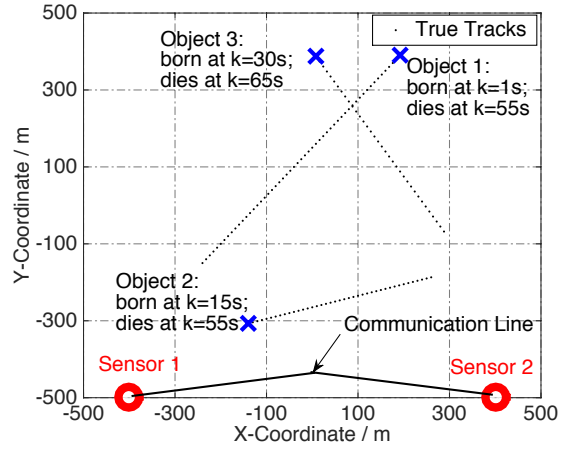

Fig. 5. The scenario of a distributed sensor network with two sensors tracking three objects.

\section{A. Scenario 1}

The robustness of the proposed R-GCI-GLMB fusion algorithm is verified by comparison with the classical GCI fusion of LMB posteriors (C-GCI-LMB) [30] in two experiments with the ABP and the PBP used respectively. To this end, we consider a scenario involving three objects on a two dimensional surveillance region $[-500,500] \mathrm{m} \times[-500,500] \mathrm{m}$, which is shown in Fig. 5 For both fusion algorithms, the LMB filter is chosen as the local filter. For GM implementations of local LMB filter and fusion algorithms, the parameters are chosen as follows: the truncation threshold for Bernoulli components is $\gamma_{t}=10^{-4}$; pruning and merging thresholds for Gaussian components are $\gamma_{p}=10^{-5}$ and $\gamma_{m}=4$, respectively; the maximum number of Gaussian components is $N_{\max }=10$. The duration of this scenario is $T=65 \mathrm{~s}$. The probability of detection $P_{D, k}$ for each sensor is 0.99 .

Experiment 1: The performance metrics for R-GCI-GLMB and C-GCI-LMB fusions in presence of an ABP are shown in Fig. 6. Specifically, the cardinality estimates (Est.) and the corresponding standard deviations (Std.) are presented in Fig. 6.a), while the OSPA errors are provided in Fig. 6(b).

Not surprisingly, C-GCI-LMB fusion completely fails (returns highly erroneous estimates) when the $\mathrm{ABP}$ is in place due to the resulting inconsistencies between label assignments in each local filters and the reliance of labeled GCI fusion on label consistency between filters. This is while R-GCIGLMB fusion leads to errors that are significantly lower than errors returned by each local filter after each transient. These results highlight the robustness of the proposed R-GCI-GLMB algorithm when label mismatches happen.

Experiment 2: In this experiment, the performance of R-GCIGLMB and C-GCI-LMB fusions are compared in presence of a non-adaptive birth model that is based on prior information PBP model [24]. The comparisons in terms of the cardinality statistics and OSPA errors between R-GCI-GLMB fusion and C-GCI-LMB fusion are presented in Figs. 7(a) and (b).

It can be seen from Fig.77(a) that cardinality estimates of the C-GCI-LMB fusion are biased with large standard deviations, while the cardinality estimates returned by the R-GCI-GLMB fusion are much more accurate with less deviations (high level of confidence). From the results shown in Fig. 7(b), we observe that whenever an object appears or disappears, the

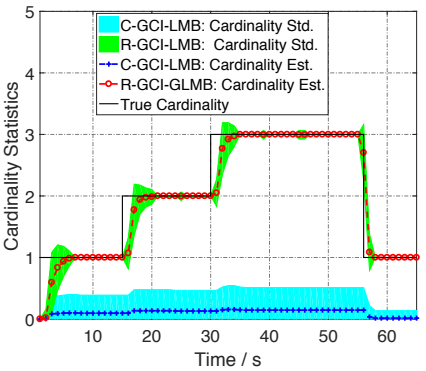

(a)

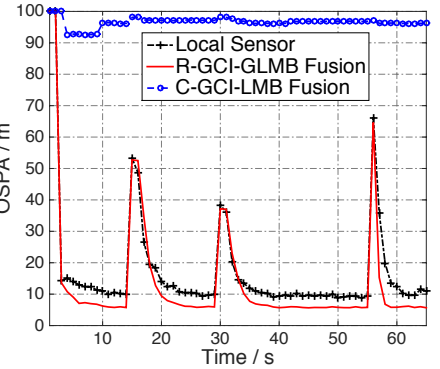

(b)
Fig. 6. Tracking performances of local sensor filter, R-GCI-GLMB and CGCI-LMB fusion algorithms in scenario 1 in presence of an adaptive birth process: (a) cardinality statistics, (b) OSPA errors.

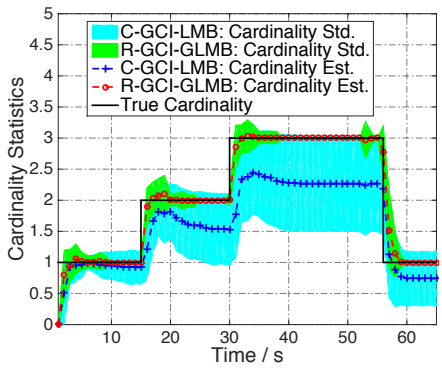

(a)

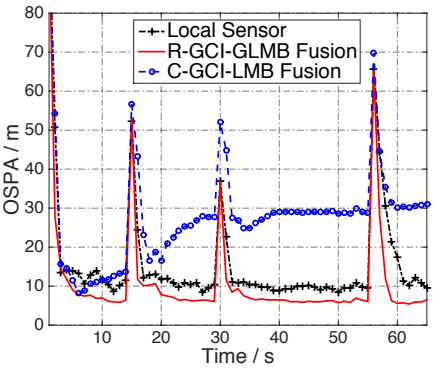

(b)
Fig. 7. Tracking performances of the local sensor filter, R-GCI-GLMB and C-GCI-LMB fusion algorithms in scenario 1 in presence of a non-adaptive birth process designed based on prior information: (a) cardinality statistics, (b) OSPA errors.

OSPA errors returned by all methods sharply increase. Another observation is while the local filter and the proposed R-GCIGLMB fusion handle the change well (and their tracking errors gradually retract after every jump), the classical GCI fusion does not survive the impact of a sudden change in number of objects (especially for the births) and its error increases. A third observation is that our R-GCI-GLMB fusion significantly outperforms the other methods in terms of the OSPA error.

The above observations are in line with the result of Example 1 and the mathematical analysis presented earlier. Each time a new object is born, the average disparity between the label information embedded in various labeled posteriors is enhanced because one more object may have different estimated labels in different sensors, which leads to a larger label inconsistency indicator $d_{G}(\boldsymbol{\Pi})$ in turn resulting in a degraded performance for C-GCI-LMB fusion.

\section{B. Scenario 2}

In order to further demonstrate the performance of the proposed R-GCI-GLMB fusion in challenging scenarios, a sensor network scenario with three sensors and eight objects is considered as shown in Fig. 8(a). The objects appear and disappear at different times as listed in Table I

The performance of the R-GCI-GLMB fusion is compared to the GCI-CPHD fusion [6], [7]. The CPHD filter and the LMB filter are chosen as the local filter for GCICPHD fusion and R-GCI-GLMB fusion, respectively. Since 


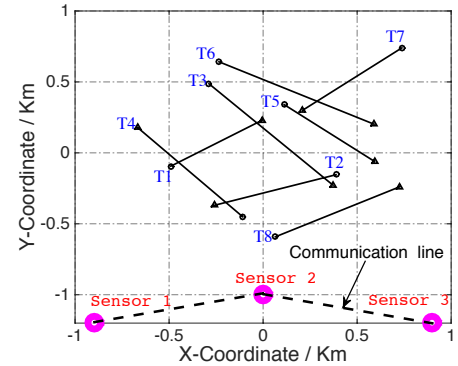

(a)

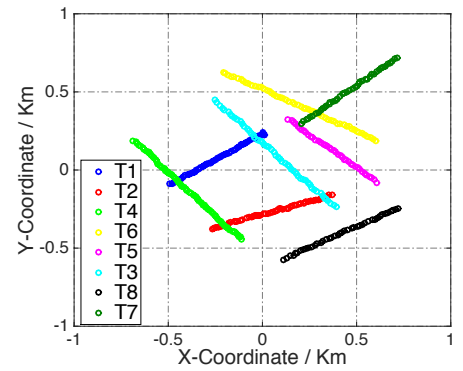

(b)
Fig. 8. (a) Scenario 2: a distributed sensor network involving three sensors tracking eight objects on a two dimensional surveillance region. (b) Tracking results of the R-GCI-GLMB fusion algorithm with different colors denoting different identities of objects under $P_{D, k}=0.98$.

TABLE I

TARGET BIRTH AND DEATH TIMES IN SCENARIO 2.

\begin{tabular}{cccccc}
\hline Target & Birth & Death & Target & Birth & Death \\
\hline T1 & $1 \mathrm{~s}$ & $56 \mathrm{~s}$ & T5 & $25 \mathrm{~s}$ & $66 \mathrm{~s}$ \\
T2 & $1 \mathrm{~s}$ & $56 \mathrm{~s}$ & T6 & $25 \mathrm{~s}$ & $81 \mathrm{~s}$ \\
T3 & $10 \mathrm{~s}$ & $66 \mathrm{~s}$ & T7 & $56 \mathrm{~s}$ & $>100 \mathrm{~s}$ \\
T4 & $10 \mathrm{~s}$ & $81 \mathrm{~s}$ & T8 & $56 \mathrm{~s}$ & $>100 \mathrm{~s}$ \\
\hline
\end{tabular}

the objects appear at unknown positions, LMB filters use an ABP introduced in [24] and CPHD filter uses the adaptive birth distribution introduced in [11]. Pruning and merging thresholds for GM implementations of local CPHD filter and GCI-CPHD fusion algorithms are chosen as $\gamma_{p}=10^{-5}$ and $\gamma_{m}=4$, respectively, and the maximum number of Gaussian components is $N_{\max }=30$. For the GM implementation of the R-GCI-GLMB fusion, the parameters are set to be the same as Scenario 1. The duration of this scenario is $T=100 \mathrm{~s}$.

The sensors have the same detection parameters and each sensor can only exchange posteriors with its neighbour(s). Therefore, sensors 1 and 3 perform fusion with two posteriors from sensor 2 and their local filters, and sensor 2 performs fusion with three posteriors from sensor 1 , sensor 3 and the local filter by sequentially applying the pairwise fusion twice.

Fig. 8 b) shows the estimated tracks returned by R-GCIGLMB fusion for a single run under $P_{D, k}=0.98$. It can be seen that R-GCI-GLMB fusion performs accurately and consistently for the entire scenario in the sense that it maintains locking on all tracks, estimates object positions accurately, and recognizes object identities correctly. Fig. 9.a) presents the cardinality estimates and the corresponding standard deviations returned by R-GCI-GLMB fusion and GCI-CPHD fusion algorithms at sensor 2 under $P_{D, k}=0.98$. It shows that cardinality estimates given by R-GCI-GLMB fusion are more accurate with less variations (higher level of confidence) than GCI-CPHD fusion. Note that since two objects are born at time $56 \mathrm{~s}$ and two objects die at time $56 \mathrm{~s}$ (as shown in Table I), the cardinality curves have a notch at time $56 \mathrm{~s}$.

Under $P_{D, k}=0.98$, the OSPA errors for tracking results returned by the algorithms are shown in Fig. 9(b). Further, we compute the corresponding standard deviations of OSPA errors and average the post-transient values over $200 \mathrm{MC}$ runs and 100 time steps, and the results are provided in Table II.
They demonstrate the performance difference between the RGCI-GLMB and GCI-CPHD fusions at sensor 2. OSPA errors of the R-GCI-GLMB fusion are significantly lower than the GCI-CPHD fusion with lower standard deviations after each transient. Moreover, when objects die at time $66 \mathrm{~s}$ and $81 \mathrm{~s}$, OSPA error of the R-GCI-GLMB filter retracts to a stable value much faster than the GCI-CPHD fusion method.

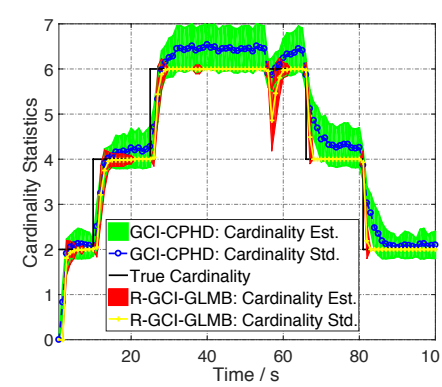

(a)

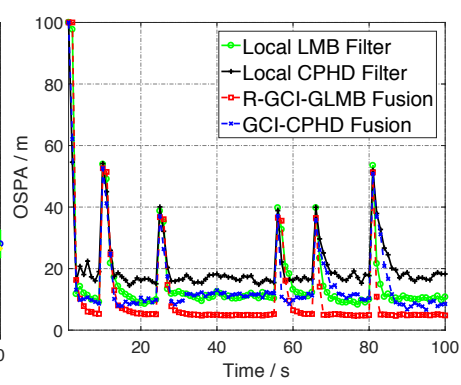

(b)
Fig. 9. Tracking performances of R-GCI-GLMB and GCI-CPHD fusion algorithms under $P_{D, k}=0.98$ in Scenario 2: (a) cardinality statistics (b) OSPA errors.

TABLE II

AVERAGE STANDARD DEVIATIONS OF OSPA ERRORS UNDER $P_{D, k}=0.98$.

\begin{tabular}{c|cccc}
\hline \hline Algorithm & R-GCI-GLMB & GCI-CPHD & Local LMB & Local CPHD \\
\hline Std. $(\mathrm{m})$ & 1.673 & 8.083 & 6.838 & 10.580 \\
\hline
\end{tabular}

To assess the computational efficiency of the algorithms, the average execution times of the R-GCI-GLMB (adopting the efficient implementation strategy) and GCI-CPHD fusions under $P_{D, k}=0.98$ are depicted in Fig. 10. It can be seen that the execution time of the R-GCI-GLMB fusion is only slightly longer than the GCI-CPHD fusion with the R-GCI-GLMB fusion providing the enhanced performance (as demonstrated previously) and also automatically accounts for track labelling.

Further, we assess the performance of R-GCI-GLMB and GCI-CPHD fusion methods under different $P_{D, k}$ values in terms of the averaged post-transient values of OSPA errors (over $200 \mathrm{MC}$ runs and 100 time steps) as shown in Table III Not surprisingly, while the performances of both algorithms degrade as the $P_{D, k}$ value decreases, the R-GCI-GLMB fusion performs remarkably better than the GCI-CPHD fusion under each $P_{D, k}$ value with the performance difference stable.

TABLE III

AVERAGE OSPA ERRORS (M) OF R-GCI-GLMB AND GCI-CPHD FUSIONS UNDER DIFFERENT $P_{D, k}$ VALUES.

\begin{tabular}{c|ccc}
\hline \hline$P_{D, k}$ & 0.98 & 0.88 & 0.78 \\
\hline R-GCI-GLMB & 5.160 & 6.026 & 7.688 \\
GCI-CPHD & 11.079 & 12.411 & 13.659 \\
\hline
\end{tabular}

To demonstrate how the performance advantage gained from sensor fusion increases with the number of sensors, we 


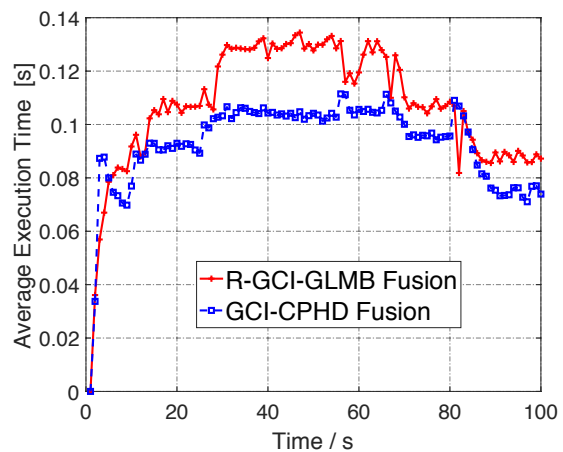

Fig. 10. Average execution times for R-GCI-GLMB and GCI-CPHD fusion algorithms under $P_{D, k}=0.98$ in Scenario 2 .

compute the OSPA errors returned by R-GCI-GLMB fusion and average the post-transient values over $200 \mathrm{MC}$ runs and 100 time steps. Table IV shows the recorded values in presence of one, two and three sensors. The results demonstrate the efficacy of the proposed sensor fusion algorithm in the sense that estimation accuracy improves with more sensors. These

TABLE IV

Average OSPA Errors $v s$ No. OF Sensors $\left(P_{D, k}=0.98\right)$

\begin{tabular}{lccc}
\hline Number of sensors & One & Two & Three \\
\hline OSPA Errors $(\mathrm{m})$ & 10.711 & 5.948 & 5.160 \\
\hline
\end{tabular}

results demonstrate that the two-step approximation used in the derivation of GCI fusion with GMB distributions is reasonable, and the significant enhancement in performance (in terms of OSPA errors) also verifies the robustness and effectiveness of the R-GCI-GLMB fusion devised and presented in this work.

\section{CONCLUSION}

In this paper, we addressed the problem of distributed multiobject tracking with labeled set filters based on generalized Covariance Intersection (GCI). Firstly, we showed that the performance of GCI fusion with labeled multi-object densities is highly sensitive to inconsistencies between label information from the local labeled posteriors. We provided a mathematical analysis from the perspective of Principle of Minimum Discrimination Information and yes-object probability. Secondly, inspired by the analysis, a novel and general solution was proposed for distributed fusion of labeled multi-object posteriors that is robust to label inconsistencies between different sensor node posteriors. Thirdly, for the case of fusing generalized labeled multi-Bernoulli (GLMB) filter family including the GLMB, $\delta$-GLMB, marginalized $\delta$-GLMB and labeled multiBernoulli (LMB) filters, we formulated the robust fusion solution. Simulation results for Gaussian mixture (GM) implementation demonstrated the robustness and effectiveness of the proposed fusion algorithms in challenging tracking scenarios.

\section{APPENDIX A}

PROOF OF PROPOSITION 2

According to (17), the unlabeled version of a GLMB density of form (4) is distributed according to

$$
\begin{aligned}
& \pi\left(\left\{x_{1}, \cdots, x_{n}\right\}\right)=\sum_{\left(\ell_{1}, \cdots, \ell_{n}\right) \in \mathbb{L}^{n}} \boldsymbol{\pi}\left(\left\{\left(x_{1}, \ell_{1}\right), \cdots,\left(x_{n}, \ell_{n}\right)\right\}\right) \\
= & \sum_{\left(\ell_{1}, \cdots, \ell_{n}\right) \in \mathbb{L}^{n}} \sum_{c \in \mathbb{C}} w^{(c)}\left(\left\{\ell_{1}, \cdots, \ell_{n}\right\}\right) \prod_{i=1}^{n} p^{(c)}\left(x_{i}, \ell_{i}\right) \\
= & \sum_{\sigma} \sum_{I \in \mathcal{F}_{n}(\mathbb{L})} \sum_{c \in \mathbb{C}} w^{(c)}(I) \prod_{i=1}^{n} p^{(c)}\left(x_{\sigma(i)}, I^{v}(i)\right) .
\end{aligned}
$$

where $\sigma$ denotes one permutation of $I, \sigma(i)$ denotes the $i$ th element of the permutation, and $I^{v}$ denotes a vector constructed by sorting the elements of the set $I$.

Let

$$
\begin{aligned}
w^{(I, c)} & \triangleq w^{(c)}(I), I \in \mathcal{F}(\mathbb{L}) \\
p^{(c), \ell}(x) & \triangleq p^{(c)}(x, \ell), \ell \in \mathbb{L} .
\end{aligned}
$$

Equation (79) can be further represented as

$$
\pi\left(\left\{x_{1}, \cdots, x_{n}\right\}\right)=\sum_{\sigma} \sum_{(I, c) \in \mathcal{F}_{n}(\mathbb{L}) \times \mathbb{C}} w^{(I, c)} \prod_{i=1}^{n} p^{(c), I^{v}(i)}\left(x_{\sigma(i)}\right)
$$

\section{APPENDIX B}

\section{ProOF OF Proposition 3}

According to (17), the unlabeled version of an LMB density of form 6 is distributed according to

$$
\begin{aligned}
& \pi\left(\left\{x_{1}, \cdots, x_{n}\right\}\right)=\sum_{\left(\ell_{1}, \cdots, \ell_{n}\right) \in \mathbb{L}^{n}} \boldsymbol{\pi}\left(\left\{\left(x_{1}, \ell_{1}\right), \cdots,\left(x_{n}, \ell_{n}\right)\right\}\right) \\
= & \sum_{\left(\ell_{1}, \cdots, \ell_{n}\right) \in \mathbb{L}^{n}} w\left(\left\{\ell_{1}, \cdots, \ell_{n}\right\}\right) \prod_{i=1}^{n} p\left(x_{i}, \ell_{i}\right) \\
= & \sum_{\sigma} \sum_{I \in \mathcal{F}_{n}(\mathbb{L})} w(I) \prod_{i=1}^{n} p\left(x_{\sigma(i)}, I^{v}(i)\right) .
\end{aligned}
$$

Let

$$
\begin{aligned}
w^{(I)} & \triangleq w^{(I)}, I \in \mathcal{F}(\mathbb{L}) \\
p^{(c), \ell}(x) & \triangleq p^{(\ell)}(x), \ell \in \mathbb{L} .
\end{aligned}
$$

Equation 81 can be further represented as

$$
\pi\left(\left\{x_{1}, \cdots, x_{n}\right\}\right)=\sum_{\sigma} \sum_{I \in \mathcal{F}_{n}(\mathbb{L})} w^{(I)} \prod_{i=1}^{n} p^{\left(I^{v}(i)\right)}\left(x_{\sigma(i)}\right)
$$

\section{APPENDIX C PROOF OF PROPOSITION 4}

Combination of (61) and (74) yields

$\boldsymbol{\pi}_{\omega}\left(\left\{\left(x_{1}, \ell_{1}\right), \cdots,\left(x_{n}, \ell_{n}\right)\right\}\right)=$

$\frac{\left[\bar{w}_{1}\left(\left\{\ell_{1}, \cdots, \ell_{n}\right\}\right)\right]^{\omega_{1}} \prod_{i=1}^{n}\left[\bar{p}_{1}^{\left(\ell_{i}\right)}\left(x_{i}\right)\right]^{\omega_{1}}}{\sum_{\left(\ell_{1}, \cdots, \ell_{n}\right) \in \mathbb{L}_{1}^{n}}\left[\bar{w}_{1}\left(\left\{\ell_{1}, \cdots, \ell_{n}\right\}\right)\right]^{\omega_{1}} \prod_{i=1}^{n}\left[\bar{p}_{1}^{\left(\ell_{i}\right)}\left(x_{i}\right)\right]^{\omega_{1}}} \times$

$\frac{1}{C} \prod_{s=1,2} \sum_{\sigma_{s}} \sum_{\mathcal{I}_{s} \in \mathcal{F}_{n}\left(\mathbb{I}_{s}\right)}\left[\widetilde{w}_{s}^{\left(\mathcal{I}_{s}\right)}\right]^{\omega_{s}}\left[\prod_{i=1}^{n} \tilde{p}_{s}^{\left(\mathcal{I}_{s}^{v}(i)\right)}\left(x_{i}\right)\right]^{\omega_{s}}$. 
As shown in Proposition 2, the unlabeled version of GLMB density in (69) is a GMB density with $\mathbb{I}_{s_{0}}=\mathbb{L}_{s_{0}}$ and $\Phi_{s_{0}}=$ $\mathbb{C}_{s_{0}}$. Hence, (71) and (72) (with $s_{0}=1$ ) can be rewritten as

$$
\begin{gathered}
\bar{r}_{1}^{(\ell)}=\sum_{\mathcal{I} \in \mathcal{F}\left(\mathbb{I}_{1}\right)} \sum_{\phi \in \Phi_{1}} 1_{\mathcal{I}}(\ell) w_{1}^{(\mathcal{I}, \phi)}=\widetilde{r}_{1}^{(\ell)} \\
\bar{p}_{1}^{(\ell)}(x)=\frac{1}{\widetilde{r}_{1}^{(\ell)}} \sum_{\mathcal{I} \in \mathcal{F}\left(\mathbb{I}_{1}\right)} \sum_{\phi \in \Phi_{1}} 1_{\mathcal{I}}(\ell) w_{1}^{(\mathcal{I}, \phi)} p_{1}^{(\phi), \ell}(x)=\widetilde{p}_{1}^{(\ell)}(x)
\end{gathered}
$$

where $\widetilde{r}_{1}^{(\ell)}$ and $\widetilde{p}_{1}^{(\ell)}(x)$ are shown in 57 , and 58 respectively. As a result, we have the following equality,

$$
\begin{aligned}
& \sum_{\left(\ell_{1}, \cdots, \ell_{n}\right) \in \mathbb{L}_{1}^{n}}\left[\bar{w}_{1}\left(\left\{\ell_{1}, \cdots, \ell_{n}\right\}\right)\right]^{\omega_{1}} \prod_{i=1}^{n}\left[\bar{p}_{1}^{\left(\ell_{i}\right)}\left(x_{i}\right)\right]^{\omega_{1}}= \\
& \sum_{\sigma} \sum_{\mathcal{I}_{1} \in \mathcal{F}_{n}\left(\mathbb{I}_{1}\right)}\left[\widetilde{w}_{1}^{\left(\mathcal{I}_{1}\right)}\right]^{\omega_{1}} \prod_{i=1}^{n}\left[\widetilde{p}_{1}^{\left(\mathcal{I}_{1}^{v}(i)\right)}\left(x_{\sigma(i)}\right)\right]^{\omega_{1}}
\end{aligned}
$$

Substitution of 84 , 857 and 86 into 83 , we have

$$
\begin{aligned}
& \pi_{\omega}\left(\left\{\left(x_{1}, \ell_{1}\right), \cdots,\left(x_{n}, \ell_{n}\right)\right\}\right) \\
= & \frac{1}{C}\left[\widetilde{w}_{1}^{\left(\left\{\ell_{1}, \cdots, \ell_{n}\right\}\right)}\right]^{\omega_{1}} \prod_{i=1}^{n}\left[\widetilde{p}_{1}^{\left(\ell_{i}\right)}\left(x_{i}\right)\right]^{\omega_{1}} \times \\
& \sum_{\sigma} \sum_{\mathcal{I}_{2} \in \mathcal{F}_{n}\left(\mathbb{I}_{2}\right)}\left[\widetilde{w}_{2}^{\left(\mathcal{I}_{2}\right)}\right]^{\omega_{2}} \prod_{i=1}^{n}\left[\widetilde{p}_{2}^{\left(\mathcal{I}_{2}^{v}(i)\right)}\left(x_{\sigma(i)}\right)\right]^{\omega_{2}}
\end{aligned}
$$

Substitution of 63, 64), 625 and (65) into (87), and utilizing Definition 5, we can obtain

$$
\begin{aligned}
& \pi_{\omega}\left(\left\{\left(x_{1}, \ell_{1}\right), \cdots,\left(x_{n}, \ell_{n}\right)\right\}\right) \\
= & \sum_{\tau \in \mathcal{T}\left(\left\{\ell_{1}, \cdots, \ell_{n}\right\}\right)} \frac{1}{C}\left[\widetilde{w}_{1}^{\left(\left\{\ell_{1}, \cdots, \ell_{n}\right\}\right)}\right]^{\omega_{1}}\left[\widetilde{w}_{2}^{\left(\tau\left(\left\{\ell_{1}, \cdots, \ell_{n}\right\}\right)\right.}\right]^{\omega_{2}} \times \\
& \prod_{i=1}^{n} \eta_{\omega}^{(\tau), \ell_{i}} \prod_{i=1}^{n} p_{\omega}^{(\tau), \ell_{i}}\left(x_{i}\right) \\
= & \sum_{\tau \in \mathcal{T}\left(\left\{\ell_{1}, \cdots, \ell_{n}\right\}\right)} w_{\omega}^{\left(\left\{\ell_{1}, \cdots, \ell_{n}\right\}, \tau\right)} \prod_{i=1}^{n} p_{\omega}^{(\tau), \ell_{i}}\left(x_{i}\right)
\end{aligned}
$$

Using the definition of $p_{\omega}^{(\tau)}(\cdot, \ell)$ in 77 and $w_{\omega}^{(\tau)}(I)$ in 78 , we can obtain (76).

\section{REFERENCES}

[1] C.-Y. Chong, S. Mori, and K.-C. Chang, "Distributed multitarget multisensor tracking," Multitarget-multisensor tracking: Advanced applications, vol. 1, pp. 247-295, 1990.

[2] R. P. Mahler, "Optimal/robust distributed data fusion: a unified approach," in Proc. SPIE Defense and Security Symp., 2000, pp. 128-138.

[3] M. Hurley, "An information-theoretic justification for covariance intersection and its generalization," in Proc. IEEE Int. Fusion Conf., July 2002, pp. 7-11.

[4] S. J. Julier, T. Bailey, and J. K. Uhlmann, "Using exponential mixture models for suboptimal distributed data fusion," in Proc. IEEE Nonlinear Statist. Signal Process. Workshop (NSSPW'6), Cambridge, U. K., 2006, pp. $160-163$.

[5] D. E. Clark, S. J. Julier, R. Mahler, and B. Ristic, "Robust multiobject sensor fusion with unknown correlations," in Sens. Signal Process. Defence (SSPD'10), Sep. 2010, pp. 1-5.

[6] M. Uney, D. E. Clark, and S. J. Julier, "Distributed fusion of PHD filters via exponential mixture densities," IEEE J. Sel. Topics Signal Process., vol. 7, no. 3, pp. 521-531, 2013.

[7] G. Battistelli, L. Chisci, C. Fantacci, A. Farina, and A. Graziano, "Consensus CPHD filter for distributed multitarget tracking." IEEE J. Sel. Topics Signal Process., vol. 7, no. 3, pp. 508-520, 2013.

[8] G. Battistelli, L. Chisci, C. Fantacci, A. Farina, and R. Mahler, "Distributed fusion of multitarget densities and consensus PHD/CPHD filters," in Proc. SPIE Defense, Security and Sensing, vol. 9474, Baltimore, MD, 2015.
[9] J. K. Uhlmann, "Dynamic map building and localization for autonomous vehicles," Unpublished doctoral dissertation, Oxford University, vol. 36, 1995.

[10] B.-N. Vo and W.-K. Ma, "The Gaussian mixture probability hypothesis density filter," IEEE Trans. Signal Process., vol. 54, no. 11, pp. 40914104, 2006.

[11] B. Ristic, D. E. Clark, B.-N. Vo, and B.-T. Vo, "Adaptive target birth intensity for PHD and CPHD filters," IEEE Trans. Aerosp. Electron. Syst., vol. 48, no. 2, pp. 1656-1668, 2012.

[12] D. Franken, M. Schmidt, and M. Ulmke, ““ Spooky action at a distance" in the cardinalized probability hypothesis density filter," IEEE Trans. Aerosp. Electron. Syst., vol. 4, no. 45, pp. 1657-1664, 2009.

[13] B.-T. Vo, B.-N. Vo, and A. Cantoni, "Analytic implementations of the cardinalized probability hypothesis density filter," IEEE Trans. Signal Process., vol. 55, no. 7, pp. 3553-3567, 2007.

[14] _ - "The cardinality balanced multi-target multi-Bernoulli filter and its implementations," IEEE Trans. Signal Process., vol. 57, no. 2, pp. 409-423, 2009.

[15] B.-N. Vo, B.-T. Vo, N.-T. Pham, and D. Suter, "Joint detection and estimation of multiple objects from image observations," IEEE Trans. Signal Process., vol. 58, no. 10, pp. 5129-5141, 2010.

[16] J. Wong, B.-T. Vo, B.-N. Vo, and R. Hoseinnezhad, "Multi-Bernoulli based track-before-detect with road constraints," in Proc. IEEE Int. Fusion Conf., 2012, pp. 840-846.

[17] R. Hoseinnezhad, B.-N. Vo, and B.-T. Vo, "Visual tracking in background subtracted image sequences via multi-Bernoulli filtering," IEEE Trans. Signal Process., vol. 61, no. 2, pp. 392-397, 2013.

[18] M. B. Guldogan, "Consensus Bernoulli filter for distributed detection and tracking using multi-static doppler shifts," IEEE Signal Process. Lett., vol. 6, no. 21, pp. 672-676, 2014.

[19] B. L. Wang, W. Yi, R. Hoseinnezhad, S. Q. Li, L. J. Kong, and X. B. Yang, "Distributed fusion with multi-Bernoulli filter based on generalized Covariance Intersection," IEEE Trans. Signal Process., vol. 65, no. 1, pp. 242-255, Jan. 2017.

[20] T. C. Li, J. M. Corchado, and S. D. Sun, "On generalized covariance intersection for distributed PHD filtering and a simple but better alternative," in Proc. IEEE Int. Fusion Conf., 2017, pp. 1-8.

[21] W. Yi, M. Jiang, R. Hoseinnezhad, and B. Wang, "Distributed multisensor fusion using generalised multi-Bernoulli densities," IET Radar, Sonar \& Nevigation, vol. 11, no. 3, pp. 434-443, Mar. 2016.

[22] B. T. Vo and B. N. Vo, "Labeled random finite sets and multi-object conjugate priors," IEEE Trans. on Signal Process., vol. 61, no. 13, pp. 3460-3475, July 2013.

[23] B.-N. Vo, B.-T. Vo, and D. Phung, "Labeled random finite sets and the Bayes multi-target tracking filter," IEEE Trans. Signal Process., vol. 62, no. 24 , pp. $6554-6567,2014$

[24] S. Reuter, B.-T. Vo, B.-N. Vo, and K. Dietmayer, "The labeled multiBernoulli filter," IEEE Trans. Signal Process., vol. 62, no. 12, pp. 32463260, 2014.

[25] M. Beard, B.-T. Vo, and B.-N. Vo, "Bayesian multi-target tracking with merged measurements using labelled random finite sets," IEEE Trans. Signal Process., vol. 63, no. 6, pp. 1433-1447, 2015.

[26] C. Fantacci, B.-T. Vo, F. Papi, and B.-N. Vo, "The marginalized $\delta$-GLMB filter," arXiv preprint arXiv:1501.00926, 2015.

[27] F. Papi, B.-N. Vo, B.-T. Vo, C. Fantacci, and M. Beard, "Generalized labeled multi-Bernoulli approximation of multi-object densities," IEEE Trans. Signal Process., vol. 63, no. 20, pp. 5487-5497, 2015.

[28] S. Q. Li, W. Yi, R. Hoseinnezhad, B. L. Wang, and L. J. Kong, "Multiobject tracking for generic observation model using labeled random finite sets," IEEE Trans. Signal Process., accepted, Sep. 2017.

[29] R. P. Mahler, Advances in Statistical Multisource-Multitarget Information Fusion. Artech House, 2014.

[30] C. Fantacci, B.-N. Vo, B.-T. Vo, G. Battistelli, and L. Chisci, "Consensus labeled random finite set filtering for distributed multi-object tracking," arXiv preprint arXiv:1501.01579, 2015.

[31] B. L. Wang, W. Yi, S. Q. Li, M. R. Morelande, L. J. Kong, and X. B. Yang, "Distributed multi-target tracking via generalized multi-bernoulli random finite sets," in Proc. IEEE Int. Fusion Conf., July 2015, pp. 253-261.

[32] F. Papi, B.-N. Vo, B.-T. Vo, C. Fantacci, and M. Beard, "Generalized labeled multi-Bernoulli approximation of multi-object densities," IEEE Trans. on Signal Process., vol. 63, no. 20, pp. 5487-5497, 2015.

[33] R. Mahler, Statistical Multisource-Multitarget Information Fusion. Norwell, MA, USA: Artech House, 2007.

[34] D. Stoyan, D. Kendall, and J. Meche, Stochastic Geometry and its Applications. John Wiey \& Sons Sons, 1995. 
[35] T. Heskes, "Selecting weighting factors in logarithmic opinion pools," in Advances in Neural Information Processing Systems, Cambridge, MA, USA: MIT Press, 1998, pp. 266-272.

[36] G. E. Box and G. C. Tiao, Bayesian Inference in Statistical Analysis. John Wiley \& Sons, 2011, vol. 40.

[37] D. Schuhmacher, B.-T. Vo, and B.-N. Vo, "A consistent metric for performance evaluation of multi-object filters," IEEE Trans. Signal Process., vol. 56, no. 8, pp. 3447-3457, 2008.

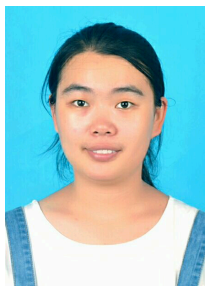

Suqi Li is born in 1990. She received the B.E. degree in electronic engineering from the University of Electronic Science and Technology of China, Chengdu, in 2011. Since September 2011, she has been pursuing the Ph.D. degree at the School of Electronic Engineering, University of Electronic Technology and Science of China. Currently, she is a visiting student with the Department of Information Engineering, University of Florence, Italy. Her research interests include random finite set, multitarget tracking, nonlinear filtering.

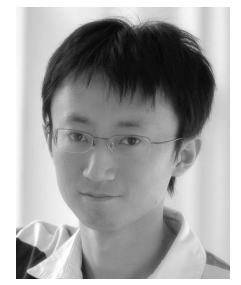

Wei Yi received the B.E. degree in electronic engineering from the University of Electronic Science and Technology of China, Chengdu, in 2006.

Since 2007, he has been pursuing the Ph.D. degree at the School of Electronic Engineering of the University of Electronic Technology and Science of China.

From March 2010 to February 2012, he was a visiting student in the Melbourne Systems Laboratory, University of Melbourne, Australia. His research interests include particle filtering and target tracking (particular emphasis on multiple target tracking and track-beforedetect techniques).

Mr. Yi received the "Best Student Paper Award" at the 2012 IEEE Radar Conference, Atlanta, United States and the "Best Student Paper Award" at the 15th International Conference on Information Fusion, Singapore, 2012.

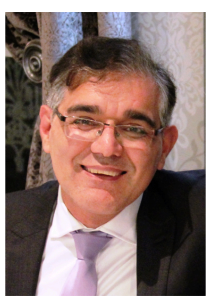

Reza Hoseinnezhad received his B.Sc., M.Sc. and Ph.D. degrees in Electronic, Control and Electrical Engineering all from the University of Tehran, Iran, in 1994, 1996 and 2002, respectively. Since 2002, he has held various academic positions at the University of Tehran, Swinburne University of Technology, the University of Melbourne and RMIT University. He is currently a senior lecturer with the School of Aerospace, Mechanical and Manufacturing Engineering, RMIT University, Victoria, Australia. His research is currently focused on development of robust estimation and visual tracking methods in a point process framework.

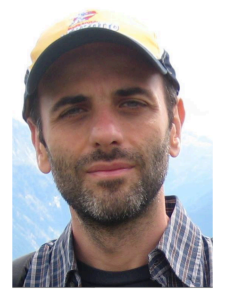

Giorgio Battistelli Giorgio Battistelli received the Laurea degree in electronic engineering and the $\mathrm{Ph} . \mathrm{D}$. degree in robotics from the University of Genoa, Genoa, Italy, in 2000 and 2004, respectively. From 2004 to 2006, he was a Research Associate with the Dipartimento di Informatica, Sistemistica e Telematica, University of Genoa. Since 2006, he has been with the University of Florence, Florence, Italy, where he is currently an Associate Professor of automatic control with the Dipartimento di Ingegneria dellInformazione. His current research interests include adaptive and learning systems, real-time control reconfiguration, linear and nonlinear estimation, hybrid systems, sensor networks, and data fusion. Dr. Battistelli was a member of the editorial boards of the IFAC Journal Engineering Applications of Artificial Intelligence and of the IEEE Transactions on Neural Networks and Learning Systems. He is currently an Associate Editor of the IFAC Journal Nonlinear Analysis: Hybrid Systems, and a member of the conference editorial boards of IEEE Control Systems Society and the European Control Association.

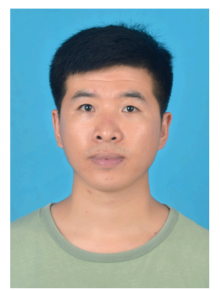

Bailu Wang received his B.S. degree from the University of Electronic Science and Technology of China (UESTC) in 2011. He is now working toward his Ph.D. degree on signal and information processing at UESTC.

From August 2016, he has been a visiting student at University of Florence, Florence, Italy. His current research interests include radar and statistical signal processing, and multi-sensor multi-target fusion.

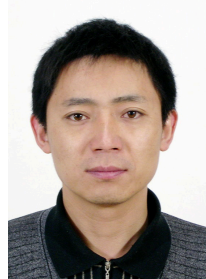

Lingjiang Kong was born in 1974. He received the B.S., M.S., and Ph.D. degrees from the University of Electronic Science and Technology of China (UESTC) in 1997, 2000 and 2003, respectively.

From September 2009 to March 2010, he was a visiting researcher with the University of Florida.

$\mathrm{He}$ is currently a professor with the School of Electronic Engineering, University of Electronic Science and Technology of China (UESTC). His research interests include multiple-input multipleoutput (MIMO) radar, through the wall radar, and statistical signal processing. 